\title{
Das Recht auf Akteneinsicht zur Prävention von menschenrechtswidriger Behandlung in der Inhaftierung
}

\section{Summary}

In order to effectively enforce the prohibition of torture, the international anti-torture conventions warrant the immediate access of the monitoring committees to the places of detention. Almost just as important is the access to their documents. Against the background of the preparation for the last visit of the European Committee for the Prevention of Torture (CPT) in Germany, the Federal Government assumed that the committee had no independent entitlement to inspect records. The contribution deals with this issue and comes to the conclusion that both from the UN Convention Against Torture (CAT) and its Optional Protocol (OPCAT), as well as from the European Convention against Torture (CPT) in Germany effective access rights to documentation arose for the prevention committees.

\section{Résumé}

Dans le but d'imposer efficacement l'interdiction de la torture, les conventions internationales contre la torture garantissent l'accès direct des commissions de contrôle aux lieux de détention. L'accès aux dossiers de ces derniers est toutefois presque aussi important. Dans le cadre des préparatifs de la dernière visite du Comité européen pour la prévention de la torture (CPT) en Allemagne, le gouvernement fédéral avait supposé que ce dernier ne bénéficiait pas d'un droit autonome à consulter les dossiers. En étudiant cette question, le présent article constate qu'il ressort tant de la Convention des Nations unies contre la torture (CAT) et de son protocole facultatif (OPCAT) que de la Convention européenne contre la torture (CPT) divers droits effectifs en Allemagne pour les comités de prévention en matière de consultation des dossiers.

Institutioneller Freiheitsentzug schafft stets Situationen mit einer latenten Anfälligkeit für Übergriffe, Misshandlungen oder Menschenrechtsverletzungen infolge unzureichender Unterbringungsbedingungen. Auch wenn die „echte“ Folter und systematische Misshandlungen einzelner Gefangener oder bestimmter Gruppen von Gefangenen in Europa nicht mehr vorzukommen scheinen, ${ }^{1}$ gibt es dennoch auch in Deutschland mit-

1 Dass der Schein mitunter trügen kann, zeigen die bis vor wenigen Jahren in mehreren EUMitgliedstaaten von der CIA im Rahmen des „War on Terror“ betriebenen Geheimgefängnisse (,Black Sites“). 
unter Anlässe sich mit Miss- und Zuständen in derartigen Institutionen zu befassen. ${ }^{2}$ Ein wichtiger Beitrag zur Zivilisierung der Gefängnisse, Gewahrsamseinrichtungen, Psychiatrien, Jugendhilfe- und Seniorenheime und anderer Orten der Freiheitsentziehung ist hierbei den Anti-Folter-Konventionen der Vereinten Nationen und des Europarates und den durch sie geschaffenen Präventionsmechanismen zuzuschreiben. Herzstück dieser Präventionsmechanismen sind Besuchskomitees, denen ein Recht auf unmittelbare Inaugenscheinnahme der Orte des Freiheitsentzuges zusteht. Ein weiteres unverzichtbares Kernelement dieser präventiven Kontrollmechanismen ist das Recht auf Einsicht in die dort geführten Unterlagen. Gerade dieses Recht hat die Bundesregierung im Vorfeld seines letzten Besuches dem European Committee on the Prevention of Torture and Inhuman or Degrading Treatment or Punishment - hierzulande besser bekannt unter seiner Abkürzung CPT - jedoch streitig gemacht. Der Beitrag befasst sich mit Begründung und Umfang eines originären Akteneinsichtsrechtes der Besuchskomitees im Rahmen der Präventionsmechanismen, die durch die Anti-Folter-Konventionen der Vereinten Nationen und des Europarates geschaffen worden sind.

\section{Keine Akteneinsicht für CPT und „Anti-Folter-Stelle“?}

Sowohl der CPT wie auch die Nationale Stelle zur Verhütung von Folter haben in ihren letzten Berichten das Thema „Akteneinsicht“ behandelt: Der CPT hat in seinem „Bericht über seinen Besuch in Deutschland vom 25. November bis 7. Dezember 2010“3 moniert, dass die Landesverwaltungen ihre Behörden im Vorfeld des Besuches so instruiert hätten, dass es keine Rechtsgrundlage für eine unmittelbare Einsichtnahme des CPT in Vollzugsakten gebe, die personenbezogene Daten der inhaftierten Personen enthielten. ${ }^{4}$ So könne das CPT in die Gefangenenpersonalakten und die Krankenakten von Gefangenen nur mit deren schriftlicher Einwilligung Einsicht nehmen. Das CPT beklagte, dass hierdurch in vielen Fällen die Einsichtnahme in Unterlagen verkompliziert und verzögert, mitunter auch verhindert worden sei. In seinem Bericht weist es auf die Wichtigkeit der unmittelbaren Einsicht in Gefangenenunterlagen hin und „empfiehlt den Bundes- und allen Landesbehörden, die Frage der Einsichtnahme in Personal- und Krankenakten seitens der Besuchsdelegationen des Ausschusses [..] zu überprüfen“".5

2 Vgl. aus jüngster Zeit bspw. die Schließung der Jugendheime der Haasenburg GmbH in Brandenburg und die im Vorfeld offenbar gewordenen Missstände, http://www.taz.de/Kinderheime-Haasenburg/!t13838/ [13.12.2013].

3 Bericht an die deutsche Regierung über den Besuch des Europäischen Ausschusses zur Verhütung von Folter und unmenschlicher oder erniedrigende Behandlung oder Strafe in Deutschland (CPT) vom 25. November bis 7. Dezember 2010 (Report to the German Government on the visit to Germany carried out by the European Committee for the Prevention of Torture and Inhuman or Degrading Treatment or Punishment (CPT) from 25 November to 7 December 2010), http://www.cpt.coe.int/en/states/deu.htm [21.10.2012].

4 CPT-Bericht (Fn 3), AbsNrn. 6-8.

5 CPT-Bericht (Fn 3), AbsNr. 8. 
Ausweislich ihrer Stellungnahme zu dem Bericht des $\mathrm{CPT}^{6}$ geht die Bundesregierung davon aus, dass es für das CPT kein Recht auf Akteneinsicht gibt. In der Stellungnahme führt die Bundesregierung aus:

„,Die Bundesregierung kann jedoch nachvollziehen, dass der CPT in dieser Rechtslage eine Einschränkung seiner Überwachungsmöglichkeiten sieht. Sie wird sich daher bemühen, im Zusammenwirken mit den Ländern zu einer befriedigenden Lösung zu kommen."

Auch die Nationale Stelle zur Verhütung von Folter thematisiert in ihrem Jahresbericht 2010/2011 die Fragestellung in Kapitel B.III „Akteneinsicht“. 8 Anlass für die Auseinandersetzung mit dem Thema sind zwei Kontrollereignisse, wobei in dem einen Fall der Nationalen Stelle die Einsicht in Gewahrsamsunterlagen verwehrt worden ist, in denen Informationen über Fixierungen vermutet wurden. In dem anderen Fall scheint hingegen weniger das Problem der Einsichtnahme in die Unterlagen im Fokus zu stehen als eine aus Sicht der Nationalen Stelle unbefriedigende Stellungnahme. Bemerkenswert ist allerdings auch, dass der Bericht Darstellungen von mehreren Besuchen in Justizvollzugsanstalten enthält und in keinem Fall von einer Verweigerung des Einblicks in Unterlagen berichtet wird, im Gegenteil wird bei vier Besuchen vermerkt, dass in Unterlagen Einblick genommen werden konnte. ${ }^{9}$ Warum - in Abweichung von der zuvor in Bezug auf das CPT dargelegten Rechtsauffassung - anscheinend der Nationalen Stelle Akteneinsicht gewährt worden ist, ergibt sich aus dem Bericht nicht.

Diese Vorgänge geben Anlass, der Frage nach dem Akteneinsichtsrecht der Kontrollinstitutionen nachzugehen. Immerhin besteht unbestritten eine völkerrechtlich verbindliche Verpflichtung der Bundesrepublik, das Europäische Übereinkommen zur Verhütung von Folter und unmenschlicher oder erniedrigender Behandlung vom 26. November 1987 (CPT), ${ }^{10}$ das Vereinte Nationen-Übereinkommen gegen Folter und andere grausame, unmenschliche oder erniedrigende Behandlung oder Strafe vom 10. Dezember 1984 (CAT) ${ }^{11}$ und das dazugehörige Fakultativprotokoll vom 18. Dezember 2002 (OPCAT) ${ }^{12}$ umzusetzen. Im Ergebnis zeigt sich, dass die eingangs geschilderte Rechtsauffassung keineswegs zwingend ist.

6 Stellungnahme der Bundesregierung zu den Empfehlungen, Kommentaren und Auskunftsersuchen des Europäischen Ausschusses zur Verhütung von Folter und unmenschlicher oder erniedrigender Behandlung oder Strafe (CPT) anlässlich seines Besuchs vom 25. November bis 7. Dezember 2010, http://www.cpt.coe.int/en/states/deu.htm [21.10.2012].

7 Stellungnahme der Bundesregierung (Fn 6), S. 3.

8 Jahresbericht 2010/2011, S. 16; http://www.antifolterstelle.de/fileadmin/dateiablage/Dokumente/Berichte/Jahresberichte/Jahresbericht2010-11.pdf [21.10.2012].

9 Dies betrifft die Besuche in den JVAen Bernau (Jahresbericht [Fn 8], S. 46), Dresden (S. 49), Chemnitz (S. 51) und Werl (S. 52).

10 European Convention for the Prevention of Torture and Inhuman or Degrading Treatment or Punishment. Die Abkürzung „CPT“ wird im Folgenden sowohl für die Europäische AntiFolter-Konvention als auch für den Ausschuss zur Verhütung von Folter verwendet.

11 Convention against Torture and Other Cruel, Inhuman or Degrading Treatment or Punishment.

12 Optional Protocol to the Convention against Torture and Other Cruel, Inhuman or Degrading Treatment or Punishment. 
Im Folgenden werden zunächst kurz der völkerrechtliche Rahmen zur Verhütung von Folter und Misshandlung bei hoheitlichem Freiheitsentzug und die darin enthaltenen Klauseln für den Informationszugang umrissen (II). Danach soll zunächst für den Strafvollzug erörtert werden, auf welcher Rechtsgrundlage eine Akteneinsicht durch die Kontrollkommissionen erfolgen kann. In diesem Rahmen wird auch der Frage nachgegangen, ob und wie die völkerrechtlichen Verpflichtungen der Europäischen Antifolterkonvention bzw. des OPCAT im innerstaatlichen Recht für den Bereich des Strafvollzugs vollzogen worden sind. Dabei wird darauf eingegangen, wie völkerrechtlich begründete gesamtstaatliche Verpflichtungen innerstaatlich vollzogen werden. Klärungsbedürftig sind sodann die jeweils anhand der konkreten Vertragsbestimmungen zu klärenden Fragen, ob diese ihrem Wesen nach unmittelbar vollziehbares Recht sein können und welche inhaltliche Reichweite sie aufweisen (III). Schließlich wird auch für andere Arten des Freiheitsentzuges kurz die rechtliche Situation beleuchtet (IV).

\section{Die völkerrechtlichen Verpflichtungen zur Prävention von Folter und Misshandlung in hoheitlichem Freiheitsentzug}

Durch die Europäische Anti-Folter-Konvention vom 26. November 1987 wurde erstmals ein internationales Kontrollsystem etabliert, indem ein Europäischer Ausschuss zur Verhütung von Folter und unmenschlicher oder erniedrigender Behandlung (CPT) geschaffen wurde, dessen Aufgabe Artikel 1 des Übereinkommens so beschreibt:

„,Der Ausschuss prüft durch Besuche die Behandlung von Personen, denen die Freiheit entzogen ist, um erforderlichenfalls den Schutz dieser Personen vor Folter und unmenschlicher oder erniedrigender Behandlung oder Strafe zu verstärken. “

Das deutsche Vertragsgesetz zu dem Europäischem Übereinkommen zur Verhütung von Folter und unmenschlicher oder erniedrigender Behandlung oder Strafe ist am 7. Dezember 1989 verkündet worden; ${ }^{13}$ das Übereinkommen ist für die Bundesrepublik am 1. Juni 1990 in Kraft getreten. ${ }^{14}$ Seit Beginn der 1990er Jahre führt das CPT seine Besuche in den Vertragsstaaten durch, in Deutschland war es das erste Mal vom 8. bis 22. Dezember 1991. ${ }^{15}$

Die zeitlich früher liegende Konvention der Vereinten Nationen war ein Meilenstein in der Ächtung der Folter, sie enthielt jedoch noch kein Element regelmäßiger und un-

13 BGB1. II v. 7.12.1989, S. 946.

14 BGBl. II v. 16.6.1990, S. 491.

15 Report to the Government of the Federal Republic of Germany on the Visit to Germany carried out by the European Committee for the Prevention of Torture and Inhuman or Degrading Treatment or Punishment from 8 to 20 December 1991, http://www.cpt.coe.int/documents/ deu/1993-13-inf-eng.pdf [4.10.2012]. 
abhängiger unmittelbarer Kontrolle an den Orten des Freiheitsentzuges. ${ }^{16}$ Ihre HauptWaffe war vor allem das öffentliche Berichtssystem im Rahmen der Vereinten Nationen. Das änderte sich jedoch nachhaltig mit dem Zusatzprotokoll zu der Vereinte $\mathrm{Na}$ tionen -Konvention, dessen Ziel es ausdrücklich ist, ,ein System regelmäßiger Besuche einzurichten, die von unabhängigen internationalen und nationalen Stellen an Orten, an denen Personen die Freiheit entzogen ist, durchgeführt werden, um Folter und andere grausame und unmenschliche oder erniedrigende Behandlung oder Strafe zu verhindern" (Art. 1 OPCAT). Als internationaler Präventionsmechanismus wird durch Art. 5 OPCAT ein „Unterausschuss zur Verhütung von Folter“ (,Subcommittee on Preventi$\left.o n^{\prime \prime}\right)$ geschaffen, dessen Aufgabe es ist, Orte der Freiheitsentziehung in den Vertragsstaaten zu besuchen und diesen Empfehlungen hinsichtlich des Schutzes von Personen, denen die Freiheit entzogen ist, vor Folter und anderer grausamer, unmenschlicher oder erniedrigender Behandlung oder Strafe zu unterbreiten (Art. 11 OPCAT).

Parallel zu dem internationalen Mechanismus, der sich erkennbar an das bereits etablierte System des CPT anlehnt, sieht das OPCAT auch die Schaffung eines unabhängigen, nationalen Präventionsmechanismus vor (Art. 17 OPCAT). Auch die Einrichtungen des nationalen Präventionsmechanismus haben gemäß Art. 19 OPCAT die Aufgabe, regelmäßig die Behandlung von inhaftierten oder untergebrachten Personen zu überprüfen und den zuständigen Behörden Empfehlungen zu unterbreiten.

Die Bundesrepublik hat das Fakultativprotokoll am 20. September 2006 unterzeichnet, durch das Vertragsgesetz vom 26. August $2008^{17}$ trat das Protokoll für Deutschland am 3. Januar 2009 in Kraft. Die daraufhin eingerichtete Nationale Stelle zur Verhütung von Folter besteht - der innerstaatlichen Verantwortlichkeit für die verschiedenen Formen des Freiheitsentzuges folgend - aus einer Bundesstelle und einer Länderkommission und ist organisatorisch in Wiesbaden bei der Kriminologischen Zentralstelle beheimatet. Die Bundesstelle ist durch Organisationserlass des Bundesjustizministeriums vom 20. November 2008 eingerichtet worden ${ }^{18}$ und hat ihre Arbeit am 1. Mai 2009 aufgenommen. Die Länderkommission wurde auf der Grundlage eines Staatsvertrags aller Bundesländer ${ }^{19}$ eingerichtet; sie hat ihre Arbeit am 24. September 2010 aufgenommen. ${ }^{20}$

Die Wirksamkeit der Kontrollmechanismen sowohl des CPT wie auch nach dem OPCAT beruhen im Wesentlichen auf zwei Elementen, nämlich dem physischen Zugang

16 Die Konvention führte einen „Ausschuss gegen Folter“ ein, dieser führt aber selbst keine Inspektionen durch, vielmehr besteht seine Aufgabe darin, die alle vier Jahre vorzulegenden Berichte der Vertragsstaaten zu analysieren und zu kommentieren. Allerdings kann der Ausschuss, wenn er zuverlässige Informationen erhalten hat, die darauf hinweisen, dass im Hoheitsgebiet eines Vertragsstaates systematische Folterungen stattfinden, nach einer Stellungnahme des Vertragsstaates eines oder mehrere seiner Mitglieder beauftragen, eine Untersuchung durchzuführen, die einen Besuch in dessen Hoheitsgebiet einschließen kann, vgl. Art. 20 CAT.

17 BGBl. II 2008, S. 854.

18 Bundesanzeiger Nr. 182 S. 4277.

19 „Staatsvertrag über die Einrichtung eines nationalen Mechanismus aller Länder nach Artikel 3 des Fakultativprotokolls vom 18. Dezember 2002 zu dem Übereinkommen der Vereinten Nationen gegen Folter und andere grausame, unmenschliche oder erniedrigende Behandlung oder Strafe" vom 1. Dezember 2009.

20 www.antifolterstelle.de/rechtsgrundlagen [15.10.2012]. 
und dem informationellen Zugang zu den Orten, an denen Personen festgehalten werden: Genauso essentiell wie der unmittelbare Eindruck durch die Besichtigung der Einrichtungen ist es für die Präventionsinstitutionen, die Unterlagen über die Inhaftierungen und Unterbringungen einsehen zu können, in denen Grund, Umfang und Ablauf der Freiheitsentzuges, besondere Vorkommnisse und die Reaktionen darauf festgehalten werden.

Das Europäische Anti-Folter-Übereinkommen bestimmt laut der amtlichen deutschen Übersetzung zum Akteneinsichtsrecht bzw. über den Zugang zu Informationen in Art. 8 Abs. 2 das Folgende:

„Eine Vertragspartei hat dem Ausschuß zur Erfüllung seiner Aufgabe folgende Erleichterungen zu gewähren: [..] d) alle sonstigen der Vertragspartei zur Verfügung stehenden Auskünfte, die der Ausschuß zur Erfüllung seiner Aufgaben benötigt. Bei Beschaffung solcher Auskünfte beachtet der Ausschuß die innerstaatlichen Rechtsvorschriften einschließlich des Standesrechts." 21

Und das OPCAT enthält die folgenden Bestimmungen über ein Informationsrecht des Unterausschusses und des nationalen Präventionsmechanismus:

Art. 14 Abs. 1: „Um dem Unterausschuss zur Verhütung von Folter die Erfüllung seines Mandats zu ermöglichen, verpflichten sich die Vertragsstaaten dieses Protokolls,

a) ihm unbeschränkten Zugang zu allen Informationen zu gewähren, welche die Anzahl der Personen, denen an Orten der Freiheitsentziehung im Sinne des Artikel 4 die Freiheit entzogen ist, sowie die Anzahl dieser Orte und ihre Lage betreffen;

b) ihm unbeschränkten Zugang zu allen Informationen zu gewähren, welche die Behandlung dieser Personen und die Bedingen ihrer Freiheitsentziehung betreffen; [..] $]^{\text {‘22 }}$

Art. 20: „Um den nationalen Mechanismen zur Verhütung von Folter die Erfüllung ihres Mandats zu ermöglichen, verpflichten sich die Vertragsstaaten dieses Protokolls, a) ihnen Zugang zu allen Informationen zu gewähren, welche die Anzahl der Personen, denen an Orten der Freiheitsentziehung im Sinne des Artikels 4 die Freiheit entzogen ist, sowie die Anzahl dieser Orte und ihre Lage betreffen;

21 ,A party shall provide the Committee with the following facilities to carry out its task: [..] d. other information available to the Party which is necessary for the Committee to carry out its task. In seeking such information, the Committee shall have regard to applicable rules of national law and professional ethics.".

22 „In order to enable the Subcommittee on Prevention to fulfill its mandate, the States Parties to the present Protocol undertake to grant it: (a) Unrestricted access to all information concerning the number of persons deprived of their liberty in places of detention as defined in article 4, as well as the number of places and their location; (b) Unrestricted access to all information referring to the treatment of those persons as well as their conditions of detention; $[. .]^{\prime \prime}$. 
b) ihnen Zugang zu allen Informationen zu gewähren, welche die Behandlung dieser Personen und die Bedingungen ihrer Freiheitsentziehung betreffen; [..] "23

Im Folgenden wird geprüft, ob bzw. wie diese völkerrechtlichen Verpflichtungen, die die Bundesrepublik eingegangen ist, im innerstaatlichen Recht vollzogen worden sind.

\section{Akteneinsichtsrecht im Strafvollzug}

Sollen die Hoheitsträger der freiheitsentziehenden Einrichtungen den Kontrollkommissionen Einblick in personenbezogene Daten gewähren, bedarf es hierfür gesetzlicher Grundlagen. Der Föderalismusreform folgend haben sich die Bundesländer BadenWürttemberg, ${ }^{24}$ Bayern ${ }^{25}$ Brandenburg, ${ }^{26}$ Hamburg, ${ }^{27}$ Hessen ${ }^{28}$ Mecklenburg-Vorpommern, ${ }^{29}$ Niedersachsen, ${ }^{30}$ Rheinland-Pfalz, ${ }^{31}$ Saarland ${ }^{32}$ und Sachsen ${ }^{33}$ zwischenzeitlich eigene Strafvollzugsgesetze gegeben, und Berlin hat den Datenschutz landes-

23 "In order to enable the national preventive mechanisms to fulfill its mandate, the States Parties to the present Protocol undertake to grant it: (a) Access to all information concerning the number of persons deprived of their liberty in places of detention as defined in article 4, as well as the number of places and their location; (b) Access to all information referring to the treatment of those persons as well as their conditions of detention [..]".

24 Gesetzbuch über den Justizvollzug in Baden-Württemberg (Justizvollzugsgesetzbuch, JVollzGB) als Artikel 1 des Gesetzes zur Umsetzung der Föderalismusreform im Justizvollzug vom 4. November 2009, GB1.2009, S. 545.

25 Gesetz über den Vollzug der Freiheitsstrafe, der Jugendstrafe und der Sicherungsverwahrung (Bayerisches Strafvollzugsgesetz - BayStVollzG) vom 10. Dezember 2007, GVB1.2007, S. 866.

26 Gesetz über den Vollzug der Freiheitsstrafe, der Jugendstrafe und der Untersuchungshaft im Land Brandenburg (Brandenburgisches Justizvollzugsgesetz - BbgJVollzG) vom 24. April 2013, GVB1. I Nr. 14/2013, S. 1.

27 Gesetz über den Vollzug der Freiheitsstrafe und der Sicherungsverwahrung (Hamburgisches Strafvollzugsgesetz - HmbStVollzG) als Artikel 1 des Gesetz zur Überarbeitung des Hamburgischen Strafvollzugsrechts und zum Erlass eines Hamburgischen Jugendstrafvollzugsgesetzes vom 14. Juli 2009, HmbGVB1.2009, S. 257.

28 Hessisches Gesetz über den Vollzug der Freiheitsstrafe und der Sicherungsverwahrung (HStVollzG) als Artikel 1 des Gesetzes zur Schaffung und Änderung hessischer Vollzugsgesetze vom 28. Juni 2010, GVB1.2010, S. 185.

29 Gesetz über den Vollzug der Freiheitsstrafe in Mecklenburg-Vorpommern (StVollzG M-V) vom 7. Mai 2013, GVBl. M-V. 2013, S. 322.

30 Niedersächsisches Justizvollzugsgesetz (NJVollzG) vom 14. Dezember 2007 (NdsGVB1. 2007, S. 720), geändert durch Gesetz vom 20.2.2009 (NdsGVBl.2009, S. 32) und Art. 2 des Gesetzes v. 25.3.2009 (NdsGVBl.2009, S. 72).

31 Landesjustizvollzugsgesetz (LJVollzG) und Landesjustizvollzugsdatenschutzgesetz (LJVollzDSG) als Artikel 1 und 3 des Landesgesetzes zur Weiterentwicklung von Justizvollzug, Sicherungsverwahrung und Datenschutz vom 8. Mai 2013, GVB1. 2013 S. 79.

32 Gesetz über den Vollzug der Freiheitsstrafe im Saarland vom 24. April 2013 (SLStVollzG), Amtsblatt 2013, S. 116.

33 Gesetz über den Vollzug der Freiheitsstrafe und des Strafarrests im Freistaat Sachsen (SächsStVollzG) vom 16. Mai 2013, SächsGVBl. 2013, S. 250. 
rechtlich durch ein Justizvollzugsdatenschutzgesetz geregelt. ${ }^{34}$ In den übrigen Bundesländern gilt das Bundes-Strafvollzugsgesetz gemäß Art. 125 a Abs. 1 GG als Bundesrecht weiter fort. Die folgende Darstellung orientiert sich an den Vorschriften des Strafvollzugsgesetzes (StVollzG).

Die Befugnisse für die Erhebung und weitere Verarbeitung personenbezogener Daten werden im StVollzG ${ }^{35}$ abschließend geregelt. ${ }^{36}$ Im Geltungsbereich dieses Gesetzes kann daher für solche Akte der Datenverwendung nicht auf Befugnisnormen außerhalb der Vollzugsgesetze zurückgegriffen werden, es muss sich eine Ermächtigung aus den bereichsspezifischen Datenschutzregelungen ergeben. ${ }^{37}$ Sowohl bei der Gewährung von Akteneinsicht als auch bei der Erteilung von Auskünften an Dritte - die weder Teil der datenführenden Stelle noch Betroffener sind ${ }^{38}$ - handelt es sich datenschutzrechtlich um einen Übermittlungsvorgang. ${ }^{39}$ Eine entsprechende Befugnis zur Übermittlung von personenbezogenen Daten ergibt sich im hiesigen Zusammenhang zunächst aus der Generalklausel des $\S 180$ Abs. 1 StVollzG in Verbindung mit der Ausübung von Kontrollbefugnissen nach $\S 180$ Abs. 3 StVollzG, § 14 Abs. 3 BDSG (s.u. 3.1). Desweiteren können die Vertragsgesetze zum CPT, zum OPCAT oder zum Staatsvertrag in Verbindung mit $\S 180$ Abs. 4 S.2 StVollzG als Ermächtigungsgrundlage herangezogen werden (s.u. 3.2).

34 Gesetz zum Schutz personenbezogener Daten im Justizvollzug und bei den Sozialen Diensten der Justiz des Landes Berlin (Justizvollzugsdatenschutzgesetz Berlin, JVollzDSG Bln) vom 21. Juni 2011 (GVB1. 2011, S. 287).

35 vgl. BT-Drs.13/10245, S. 30; Goerdeler/Weichert in: J. Feest/ W. Lesting (Hrsg.): Strafvollzugsgesetz, (Alternativ-) Kommentar, 6. Auflage, Köln 2012 (zit.: AK-Bearbeiter), § 179 Rn 6 und $\S 180$ Rn 2; Schmid in: H. D. Schwind/ A. Böhm/ J.-M. Jehle/ K. Laubenthal (Hrsg), Strafvollzugsgesetz - Bund und Länder, Kommentar, 5. Auflage, Berlin 2009 (zit.: SBJL-Bearbeiter), § $179 \mathrm{Rn} 1$ und $\S 187 \mathrm{Rn} 28$.

36 ebenso in den Straf- oder Justizvollzugsgesetzen von Bayern, Hamburg, Niedersachsen und Saarland sowie dem Berliner JVollzDSG; die übrigen Landes-Strafvollzugsgesetze sehen eine ergänzende Anwendung des Landes-DSG vor.

37 Hingegen sehen § 55 JVollzGB I BaWü und § 58 Abs. 1 HStVollzG auch die Verarbeitung personenbezogener Daten auf der Grundlage von gesetzesexternen Befugnisnormen vor: Diese beiden Gesetze sind daher hinsichtlich der Datenverarbeitung nicht abschließend (so auch ausdrücklich LT-Drs.12/1241 S.29 zum früheren § 3 JVollzDSG BaWü, der textidentisch ist mit dem jetzigen $\S 29$ JVollzGB I BaWü, LT-Drs.14/5012, S.178, und die Begründung des HStVollzG zu $\S 58$ nach Hessisches Ministerium der Justiz, für Integration und Europa (Hrsg.), Hessisches Strafvollzugsgesetz, Wiesbaden 2010, http://verwaltung.hessen. $\mathrm{de} / \mathrm{irj} /$ servlet/prt/portal/prtroot/slimp.CMReader/zentral_15/zentral_Internet/med/5ef/5ef6f9 a3-dc47-c21f-012f-31e2389e4818,22222222-2222-2222-2222-222222222222, true.pdf [30.10.2012], S. 65). Für diese Länder stellt sich daher die „Einstiegsproblematik“ über eine Befugnisnorm aus dem Vollzugsgesetz nicht - die durch die Vertragsgesetze zu den jeweiligen Abkommen geschaffenen Ermächtigungsnormen können unmittelbar herangezogen werden.

$38 \S 3$ Abs. 8 BDSG iVm $§ 187$ StVollzG; vgl. SBJL-Schmid (Fn 35), § 187 Rn 9; AKGoerdeler/Weichert (Fn 35), § 180 Rn 13.

39 AK-Goerdeler/Weichert (Fn 35), § 180 Rn 12; SBJL-Schmid (Fn ), § 180 Rn 7. 


\section{Generalklausel und Kontrollbefugnisse}

Die Generalklausel ermächtigt die Vollzugsbehörden, personenbezogene Daten zum Zwecke des Vollzugs der Freiheitsstrafe zu verarbeiten. „Verarbeiten“ beinhaltet auch die Übermittlung von vorhandenen personenbezogenen Daten (§ 3 Abs. 4 BDSG). ${ }^{40}$ Vollzugliche Zwecke sind dann gegeben, wenn die Vollzugsbehörde ihre Aufgaben ohne den jeweiligen Verarbeitungsschritt nicht sachgerecht, also nicht rechtmäßig, vollständig und in angemessener Zeit erfüllen kann. ${ }^{41}$ Es genügt hingegen nicht, dass die jeweilige Datenverarbeitung geeignet oder zweckmäßig ist. ${ }^{42}$ Die Limitierung der Befugnis zur weiteren Nutzung und Verarbeitung rechtmäßig erhobener personenbezogener Daten auf vollzugliche Zwecke ist Ausdruck des Zweckbindungsgrundsatzes. Dient die Verarbeitung nicht vollzuglichen Zwecken, kann sie nicht auf die Generalklausel gestützt werden, vielmehr ist eine spezielle Ermächtigungsnorm erforderlich.

Eine Verfolgung vollzuglicher Zwecke liegt zunächst nur vor, wenn die Verarbeitung unmittelbar für den Vollzug der Freiheitsstrafe erforderlich ist. Dies ist bei der Übermittlung von Gefangenendaten an die Präventionseinrichtungen nicht der Fall, da die Vollzugsbehörden die Freiheitsstrafen ebensogut ohne die Übermittlung durchführen können. Dass mittelbar das Vertrauen in den Vollzug gestärkt und rechtswidrige Akte im Vollzug der Freiheitsstrafe vermieden werden können, ändert an diesem Befund nichts.

Für das CPT, den Vereinten Nationen-Unterausschuss und die Nationale Stelle kommen jedoch die Wahrnehmung von Kontrollbefugnissen gemäß § 180 Abs. 3 StVollzG in Betracht: Nach dieser gesetzlichen Fiktion liegt eine Zweckänderung nicht vor bei Verarbeitungen oder Nutzungen zum Zwecke des gerichtlichen Rechtsschutzes sowie - durch Verweis auf $\S 14$ Abs. 3 BDSG - bei der Wahrnehmung von Aufsichts- oder Kontrollbefugnissen, der Rechnungsprüfung, der Durchführung einer Organisationsuntersuchung für die verantwortliche Stelle oder bei Ausbildungs- und Prüfungszwecken. Die Nutzung oder Verarbeitung der personenbezogenen Daten wird dann behandelt, als diene sie der Verfolgung vollzuglicher Zwecke, so dass die Vollzugsbehörde die rechtmäßig erhobenen Daten auf der Grundlage der Generalklausel nutzen und weiter verarbeiten darf. ${ }^{43}$ Die Bestimmung dient dem Interesse an einer funktionsfähigen und ordnungsgemäßen Verwaltung und soll dazu Kontrollen über die Rechtmäßigkeit, Zweckmäßigkeit und Kosteneffektivität ermöglichen ${ }^{44}$ Weder nach dem Wortlaut noch aufgrund der Entstehungsgeschichte ${ }^{45}$ ist die Ausübung der Kontrollbefugnisse nur auf die unmittelbar übergeordneten Aufsichtsbehörden begrenzt. ${ }^{46}$ Die „Wahrnehmung von Kontrollbefugnissen“ ist als eigenständiges Tatbestandsmerkmal zu verstehen und nicht

40 AK-Goerdeler/Weichert (Fn 35), § 180 Rn 6; SBJL-Schmid (Fn 35), § 180 Rn 4.

41 AK-Goerdeler/Weichert (Fn 35), § 180 Rn 4; SBJL-Schmid (Fn 35), § 179 Rn 5.

42 AK-Goerdeler/Weichert (Fn 35), § 180 Rn 4; SBJL-Schmid (Fn 35), § 179 Rn 5.

43 AK-Goerdeler/Weichert (Fn 35), § 180 Rn 32; SBJL-Schmid (Fn 35), § 180 Rn 20.

44 Wedde in: W. Däubler/ T. Klebe/ P. Wedde/ T. Weichert: Bundesdatenschutzgesetz, Kompaktkommentar zum BDSG, 3. Auflage, Frankfurt am Main 2010 (zit.: DKWW-Bearb), § 14 Rn 23; Schomerus in: P. Gola/ C. Klug/ B. Körffer: Bundesdatenschutzgesetz, Kommentar, 10. Auflage, München 2010 (zit.: Gola/Schomerus-Bearb., § ... Rn ...), § 14 Rn 24; SBJLSchmid (Fn 35), § 180 Rn 23.

45 vgl. die Gesetzesbegründung zu $§ 12$ Abs. 3 BDSG 1989, BT-Drs.11/4306, S.44.

46 a.A. SBJL-Schmid (Fn 35), § 180 Rn 23. 
als Teil der Aufsichtsbefugnisse. Dafür spricht auch, dass die weiteren Tatbestände des $\S 14$ Abs. 3 BDSG - Rechnungsprüfung, Organisationsprüfung - auch von anderen öffentlichen Stellen, die nicht Aufsichtsbehörden sind, und sogar von privaten Unternehmen wahrgenommen werden. Entscheidend ist, dass es um eine Kontrolle der (Vollzugs-) Behörde im Sinne einer Überprüfung ihrer Tätigkeit auf Rechtmäßigkeit und Ordnungsgemäßheit durch eine von ihr unabhängige Instanz geht, die die Behörde über festgestellte Unregelmäßigkeiten oder Verstöße informiert. Nicht unbedingt erforderlich für die Ausübung einer Kontrolle ist, dass die Kontrollinstanz über Sanktions- oder Anordnungsbefugnisse verfügt (siehe bspw. die Rechnungshöfe des Bundes und der Länder). Handelt es sich bei der Kontrolleinrichtung um eine andere öffentliche Stelle, spricht dies umso mehr dafür, eine echte Kontrollkompetenz im Sinne dieser Vorschrift anzunehmen. Abzustellen ist dabei auf die dieser Instanz durch einen innerstaatlichen Rechtsakt eingeräumten Befugnisse.

Diese Befugnisse ergeben sich - wie unten zu zeigen ist - für das CPT aus dem Vertragsgesetz zur Europäischen Antifolterkonvention, für den Vereinte Nationen-Unterausschuss zur Verhütung von Folter aus dem Vertragsgesetz zur Vereinte NationenAntifolterkonvention und für die Nationale Stelle aus den Vertragsgesetzen zum Staatsvertrag über die Einrichtung eines nationalen Mechanismus nach Artikel 3 des OPCAT vom 1. Dezember 2009. Im Folgenden soll kurz auf die Umsetzung völkerrechtlichvertraglicher Verbindlichkeiten in der innerstaatlichen Rechtsordnung eingegangen werden (a), um anschließend zunächst für das CPT zu klären, ob unmittelbar anwendbare Kontrollbefugnisse geschaffen worden sind und welchen Inhalt und Umfang sie hinsichtlich des Zugangs zu Informationen über Gefangene haben (b). Anschließend sollen dieselben Fragen für die durch das OPCAT geschaffenen Institutionen geklärt werden (c).

\section{a) Umsetzung völkerrechtlicher Verträge im innerstaatlichen Recht}

In jedem föderalen Staatswesen ist die Gestaltung der Außenvertretung (auswärtige Gewalt) im Rahmen der sog. vertikalen Gewaltenteilung zwischen Bundes- und Landesebene zu klären. Grundsätzlich muss der völkerrechtliche Vertragsabschluss und die Herstellung der Verbindlichkeit nach außen vom innerstaatlichen Vollzug der eingegangenen Verpflichtungen unterschieden werden.

\section{(1) Der Bund als primärer Inhaber der auswärtigen Vertragsabschlusskompetenz}

Die auswärtige Gewalt einschließlich des Rechts zum Abschluss völkerrechtlicher Verträge $^{47}$ steht gemäß Art. 32 Abs. 1 GG dem Bund zu. Dies kann jedoch zu Friktionen mit der innerstaatlichen Kompetenzordnung für die Gesetzgebung führen. ${ }^{48}$ Unproblematisch besteht eine (ausschließliche) Vertragskompetenz des Bundes in den Bereichen, in denen er auch innerstaatlich eine ausschließliche oder konkurrierende Gesetzge-

47 Streinz in: M. Sachs (Hrsg.), Grundgesetz, Kommentar, 6. Auflage, München 2011 (zit.: Sachs-Bearbeiter), Art. 32 Rn 10.

48 vgl. Sachs-Streinz (Fn 47), Art. 32 Rn 37. 
bungskompetenz hat. ${ }^{49}$ Umstritten und bis heute nicht abschließend geklärt ist, wie dies für Bereiche aussieht, in denen ausschließlich die Länder nach innerstaatlichem Recht die Rechtssetzungskompetenz haben, ${ }^{50}$ wie bspw. beim Polizeirecht oder seit der Föderalismusreform beim Strafvollzug: Hier steht eine föderalistische Auffassung, die die Befugnis der Länder zum Abschluss völkerrechtlicher Verträge in ,ihren“ Gesetzgebungsmaterien gemäß Art. 32 Abs. 3 GG als ausschließlich versteht, einer zentralistischen Meinung gegenüber, die diese Bestimmung im Sinne einer konkurrierenden Befugnis begreift, so dass der Bund auch dort völkerrechtliche Verträge abschließen kann, wo er nach den Art. $72 \mathrm{ff}$. GG keine Gesetzgebungskompetenz besitzt. ${ }^{51}$ Diese kompetentielle Kollisionslage versucht das „Lindauer Abkommen“ zwischen Bund und Ländern vom 14. November $1957^{52}$ aufzulösen. Es stellt hierzu einerseits fest, dass Bund und Länder an ihren entgegengesetzten Auffassungen festhalten und etabliert andererseits einen modus vivendi, nach dem der Bund vor dem Abschluss von Staatsverträgen, die Verpflichtungen des Bundes oder der Länder begründen sollen, vorab das Einverständnis der Länder einholt. ${ }^{53}$ Die Erklärung des Einverständnisses ist von der Zustimmung des Bundesrates im Rahmen des Gesetzgebungsverfahrens der Vertragsgesetze zu unterscheiden und wird von diesem nicht ersetzt. ${ }^{54}$ Faktisch besteht auf der Grundlage des Abkommens somit die Möglichkeit für den Bund, auch dort völkerrechtliche Verträge mit Verpflichtung zum Gesetzesvollzug einzugehen, wo ausschließlich die Länder die Gesetzgebungskompetenz besitzen. ${ }^{55}$

\section{(2) Die Beteiligung der Legislative am Abschluss völkerrechtlicher Verträge}

Völkerrechtliche Verträge des Bundes werden durch die Bundesregierung - in Vertretung für den Bundespräsidenten - abgeschlossen. ${ }^{56}$ Beziehen sie sich auf Gegenstände der (Bundes-) Gesetzgebung, schreibt Art. 59 Abs. 2 S. 1 GG die Beteiligung der gesetzgebenden Körperschaften und damit das sog. zweiphasige Verfahren der Ratifikation zur Herstellung der völkerrechtlichen Verbindlichkeit vor. ${ }^{57}$ Obschon Art. 59 Abs. 2 S. 1 GG von „Gegenständen der Bundesgesetzgebung“ spricht, zielt die Bestimmung nicht auf eine Abgrenzung der Verbandskompetenzen von Bund und Ländern

49 Sachs-Streinz (Fn 47), Art. 32 Rn 25.

50 vgl. Sachs-Streinz (Fn 47), Art. 32 Rn 31.

51 Darstellung beider Ansichten und ihrer Argumente bei Sachs-Streinz (Fn 47), Art. 32 Rn 32 ff. mwN.

52 Verständigung zwischen der Bundesregierung und den Staatskanzleien der Länder über das Vertragsschließungsrecht des Bundes, BullBReg 1957, S.1966, auch abgedruckt bei SachsStreinz (Fn 47), Art. 32 Rn 35 und Hillgruber in: B. Schmidt-Bleibtreu/ H. Hofmann/ A. Hopfauf (Hrsg.): Kommentar zum Grundgesetz, 12. Auflage, 2011 (zit.: SBHH-Bearbeiter), Art. 32 Rn 19.

53 Pieper in: V. Epping/ C. Hillgruber: Beck'scher Online-Kommentar Grundgesetz, Edition 16, Stand: 1.10.2012, München 2012 (zit.: Epping/Hillgruber-Bearb.), Art. 59 Rn 34; SachsStreinz (Fn 47), Art. 32 Rn 36.

54 Epping/Hillgruber-Pieper (Fn 53), Art. 59 Rn 34; Sachs-Streinz (Fn 47), Art. 32 Rn 36.

55 Sachs-Streinz (Fn 47), Art. 32 Rn 42.

56 Sachs-Streinz (Fn 47), Art. 59 Rn 14.

57 Sachs-Streinz (Fn 47), Art. 59 Rn 15. 
(Bundesgesetzgebung vs. Landesgesetzgebung), sondern auf die Abgrenzung von Gegenständen der Gesetzgebung zum bloßen Verwaltungsbereich ab. ${ }^{58} \mathrm{Ob}$ eine völkerrechtliche Vertragsnorm in diesem Sinne ein Gegenstand der Gesetzgebung ist, ist danach zu bestimmen, ob ihr Regelungsgehalt unter den verfassungsrechtlichen Vorbehalt des Gesetzes fällt. ${ }^{59}$

Die Beteiligung der Legislative erfolgt durch das Vertragsgesetz (auch Zustimmungsgesetz genannt) in der Form eines Bundesgesetzes; für das Gesetzgebungsverfahren und insbesondere für die Art der Einbindung des Bundesrates in den Formen des Zustimmungs- oder Einspruchsgesetzes gelten die normalen innerstaatlichen Regeln. ${ }^{60}$ Als Teil des Ratifizierungsverfahrens kommt dem Vertragsgesetz die Funktion einer Ratifizierungsermächtigung für die Exekutive (Bundesregierung, Bundespräsident) zu. ${ }^{61} \mathrm{Zudem}$ erfolgt so eine präventive Vollzugssicherung für völkerrechtliche Verpflichtungen, die nur durch Gesetzgebungsakte innerstaatlich vollzogen werden können: ${ }^{62}$ Denn eine weitere Funktion des Vertragsgesetzes besteht in der Überführung der Vertragsbestimmungen in innerstaatlich geltendes bzw. anzuwendendes Recht (Transformationsfunktion). ${ }^{63}$

\section{(3) Die Umsetzung von Völkervertragsrecht in innerstaatliches Recht}

Für den Weg, über den völkerrechtliches (Vertrags-) Recht zu innerstaatlich anzuwendendem Recht wird, werden im Wesentlichen zwei Erklärungen vertreten:

- Nach der Transformationstheorie, die der dualistischen Auffassung von getrennten Sphären des Völker- und des nationalen Rechts nahe steht, ${ }^{64}$ überführt das Vertragsgesetz eine völkerrechtliche Norm aus der Sphäre des Völkerrechts in das innerstaatliche Recht. Das Vertragsgesetz wird so zu einer von der nach außen gerichteten völkerrechtlichen Verpflichtung des Staatswesens getrennten, eigenständigen innerstaatlichen Rechtsquelle. ${ }^{65}$

- Nach der Vollzugstheorie, die auch mit der monistischen Auffassung einer einheitlichen Rechtssphäre kompatibel ist, ${ }^{66}$ erteilt das Vertragsgesetz dagegen einen inner-

58 BVerfGE 1, 372, 388 = NJW 1952, 970; Sachs-Streinz (Fn 47), Art. 59 Rn 31; Epping/Hillgruber-Pieper (Fn 53), Art. 59 Rn 30.

59 BVerfGE 1, 372, 388 = NJW 1952, 970; 48, 89, $126 \mathrm{f}=$ NJW 1978, 2385; 77, 170, $231=$ NJW 1988, 1651; Sachs-Streinz (Fn 47), Art. 59 Rn 26; Epping/Hillgruber-Pieper (Fn 53), Art. 59 Rn.27.

60 BVerfGE, 1, 372, 411 = NJW 1952, 970; 8, 274, 294 f; Epping/Hillgruber-Pieper (Fn 53), Art. 59 Rn 35 \& 38; SBHH-Butzer/Haas (Fn 52), Art. 59 Rn 97.

61 Epping/Hillgruber-Pieper (Fn 53), Art. 59 Rn 36; SBHH-Butzer/Haas (Fn 52), Art. 59 Rn 109.

62 BVerfGE 90, 286, 357; 58, 1, 37; 104, 151, 209; 118, 244, 258; Becker, Völkerrechtliche Verträge und parlamentarische Gesetzgebungskompetenz, NVwZ 2005, 289; SBHH-Butzer/ Haas (Fn 52), Art. 59 Rn 59.

63 BVerfGE NJW 2004, 3407, 3408; Becker, NVwZ 2005, 289; SBHH-Butzer/Haas (Fn 52), Art. $59 \mathrm{Rn} 60$.

64 vgl. SBHH-Butzer/Haas (Fn 52), Art. 59 Rn $111 \mathrm{mwN}$.

65 vgl. Becker (Fn 62), NVwZ 2005, 289; SBHH-Butzer/Haas (Fn 52), Art. 59 Rn 112 mwN.

66 vgl. Becker (Fn 62), NVwZ 2005, 289, 290. 
staatlichen Vollzugs- oder Rechtsanwendungsbefehl für die Vertragsnormen, die ihren völkerrechtlichen Charakter beibehalten. ${ }^{67}$

Für die Auslegung der Vertragsnormen ergeben sich dementsprechend (zunächst) unterschiedliche Ansatzpunkte: ${ }^{68}$ Da nach der Vollzugstheorie der völkerrechtliche Charakter der Vertragsnormen erhalten bleibt, erfolgt ihre Auslegung auch nach völkerrechtlichen Grundsätzen (insbesondere nach Art. 31-33 des Wiener Übereinkommens über das Recht der Verträge (WVK)). Hingegen sieht die Transformationstheorie in dem völkerrechtlichen Vertrag und seinem Vertragsgesetz zwei voneinander getrennte Rechtsquellen, daher gelten nach ihr für die Auslegung des Vertragsgesetzes die nationalstaatlichen Auslegungsmethoden. ${ }^{69}$ Allerdings wird auch nach diesem Ansatz die Genese des Vertragsgesetzes nicht außer Betracht bleiben können, so dass auf diesem Wege auch der völkerrechtliche Gehalt des Vertrages in die Auslegung einfließen muss. Das Bundesverfassungsgericht hat sich in seiner früheren Rechtsprechung eher an der Transformationslehre orientiert, ${ }^{70}$ spricht in seinen neueren Entscheidungen jedoch häufig von einem innerstaatlichen Rechtsanwendungsbefehl. ${ }^{71}$ Für die Auslegung von Vertragsgesetzen greift es auf völkerrechtliche Gesichtspunkte zurück und betont die Völkerrechtsfreundlichkeit des Grundgesetzes. ${ }^{72}$

Das Vertragsgesetz unterliegt denselben formellen und materiellen Rechtmäßigkeitsvoraussetzungen wie „,normales“ innerstaatliches Recht. ${ }^{73}$ Daher richtet sich die Transformationskompetenz nach der Gesetzgebungskompetenzordnung des Grundgesetzes, so dass im Bereich der ausschließlichen Landesgesetzgebung die Umsetzung der Vertragsnormen Sache der Länder ist ${ }^{74}$ (dennoch bedarf es im Rahmen der Ratifizierung eines Bundes-Vertragsgesetzes, um die parlamentarische Mitwirkung der Legislative gemäß Art. 59 Abs. 2 S. 1 GG sicher zu stellen).

\section{(4) Unmittelbare Anwendbarkeit des Vertragsgesetzes}

Schließlich ist von der Transformation bzw. der Transformationskompetenz die Frage der unmittelbaren Vollziehbarkeit einer Vertragsnorm zu trennen. Ob eine Vertragsnorm direkt anwendbar ist (sog. self-executing-Norm) und unmittelbar Rechte und Pflichten für Bürger und Hoheitsträger begründet, ist durch Auslegung des Vertrages zu ermitteln und hängt von seiner Ausrichtung und der inneren Qualität der Bestimmung ab. Entscheidend ist, ob die Bestimmung nach Inhalt, Zweck und Fassung so hinreichend bestimmt ist, dass sie ohne weitere Rechtssetzungsakte vom Adressaten angewendet

67 vgl. Becker (Fn 62), NVwZ 2005, 289; SBHH-Butzer/Haas (Fn 52), Art. 59 Rn 112.

68 ebenso gilt dies für Fragen des innerstaatlichen Rangs der Vertragsnormen, des innerstaatlichen Inkrafttretens sowie der Beendigung und Veränderung des Vertrages, SBHH-Butzer/ Haas (Fn 52), Art. 59 Rn 114.

69 SBHH-Butzer/Haas (Fn 52), Art. 59 Rn 114.

70 BVerfGE 1, 396, $410 \mathrm{f}=$ NJW 1952, 970; 42, 263, $284=$ NJW 1976, 1783.

71 BVerfGE 104, 151, 209 = NJW 2002, 1559; 128, 326, 367; beide Begrifflichkeiten: BVerfGE 111, 307, $316 \mathrm{f}$. = NJW 2004, 3407.

72 BVerfGE 111, 307, $316 \mathrm{f}$. = NJW 2004, 3407; 128, 326, 367 f; vgl. SBHH-Butzer/Haas (Fn 52), Art. $59 \mathrm{Rn} 118 \mathrm{mwN}$.

73 Epping/Hillgruber-Pieper (Fn 53), Art. 59 Rn 48.

74 Epping/Hillgruber-Pieper (Fn 53), Art. 59 Rn 34; SBHH-Hillgruber (Fn 52), Art. 32 Rn 18. 
werden kann. ${ }^{75}$ Non-self-executing sind Normen, bei denen der Vertrag selbst bestimmt oder es sich aus dem Regelungszusammenhang ergibt, dass sie nicht unmittelbar anwendbar sein sollen, und solche, die weiterer konkretisierender Rechtssetzungsakte bedürfen, um angewendet werden zu können.

Für die Frage, ob durch die Vertragsgesetze zum CPT, zum OPCAT oder zum Staatsvertrag über die Einrichtung der Länderkommission unmittelbar anwendbare Rechtsgrundlagen für ein Akteneinsichtsrecht der jeweiligen Kontrollgremien geschaffen worden sind, kommt es somit auf drei Aspekte an: 1. auf die Gesetzgebungskompetenz zum Erlass einer entsprechenden Regelung, 2. auf die Eigenschaft der jeweiligen Vertragsnorm als self-executing und 3. auf den konkreten, ggf. durch Auslegung zu ermittelnden Inhalt der Vertragsnorm.

\section{b) Art. 8 Abs. 2 CPT-VertrG als Rechtsgrundlage für einen Informationszugang im} Strafvollzug

\section{(1) Gesetzgebungskomptenz}

Bei Verabschiedung und Inkrafttreten des Vertragsgesetzes zum CPT war eine Gesetzgebungskompetenz des Bundes für den Bereich Strafvollzug gegeben: Das Vertragsgesetz trat am 8. Dezember 1989 in Kraft. ${ }^{76} \mathrm{Zu}$ diesem Zeitpunkt war der Kompetenztitel „Strafvollzug“ noch gemäß Art. 74 Abs. 1 Nr. 1 GG Gegenstand der konkurrierenden Gesetzgebungskompetenz des Bundes. Damit steht einem wirksamen Zustandekommen der im CPT-Vertragsgesetz (VertrG) vorgesehenen Kontrollkompetenzen nichts entgegen.

Für alle Länder, in denen nach der Föderalismusreform das StVollzG gemäß Art. 125 a Abs. 1 GG als partikulares Bundesrecht fortgilt, gilt somit auch das CPTVertrG fort.

Für die Länder Baden-Württemberg, Bayern, Brandenburg, Hamburg, Hessen, Mecklenburg-Vorpommern, Niedersachsen, Rheinland-Pfalz, Saarland und Sachsen, die nach der Föderalismusreform eigene Gesetze für den Strafvollzug erlassen haben, ${ }^{77}$ und für Berlin, das durch sein Justizvollzugsdatenschutzgesetz (JVollzDSG) ${ }^{78}$ eine Teilersetzung des vollzuglichen Datenschutzrechtes vorgenommen hat, ist hingegen zu klären, ob das CPT-VertrG für den Bereich Strafvollzug seine Gültigkeit verloren hat, weil es durch Landesrecht ersetzt worden ist. Da Teilersetzungen des fortgeltenden Bundesrechtes möglich sind, ${ }^{79}$ bedeutet auch die Schaffung eines Landesgesetzes für den Vollzug der Freiheitsstrafe oder sogar eines Gesetzes, das mehrere Vollzugsbereiche zusammenfassend regelt, nicht zwangsläufig, dass das neue Landes-Vollzugsrecht das

75 BVerfGE 29, 348, 360; BVerwGE 80, 233, 235; 87, 11, 13; SBHH-Butzer/Haas (Fn 52), Art. 59 Rn 119 f; Sachs-Streinz (Fn 47), Art. 59 Rn 69; Epping/Hillgruber-Pieper (Fn 53), Art. $59 \mathrm{Rn} 40$.

76 Nach Art. 3 Abs. 1 VertrG am Tage nach der Verkündung, die durch BGBl.II v. 7.12.1989 erfolgt ist.

77 S. Fn 24 bis 33.

78 S. Fn 34.

79 BVerfGE 111, 10, 30; Sachs-Degenhart (Fn 47), Art. 125 a Rn 6; SBHH-Maiwald (Fn 52), Art. 125 a Rn 6. 
gesamte Bundes-Vollzugsrecht unter Einschluss des CPT-VertrG ersetzt. Ob die bundesrechtlichen Kontrollkompetenzen des CPT durch Landesrecht ersetzt werden sollten, ist durch Auslegung der Landes-Vollzugsgesetze zu klären. Da letztlich kein Land die Stellung des CPT und seines Informationszugangs in den Vollzugsgesetzen regelt oder die Kontrollen durch das CPT grundsätzlich in Frage gestellt hat, spricht dies für eine Nicht-Ersetzung und Fortgeltung des Vertragsgesetzes.

\section{(2) Self-Executing or not?}

Art. 8 Abs. 2 lit.d CPT verpflichtet nach der amtlichen deutschen Übersetzung zunächst die Vertragspartei, dem Ausschuss alle zur Erfüllung seiner Aufgaben erforderlichen Auskünfte zu gewähren. Damit wird einerseits der Ausschuss als Rechtsinhaber ausdrücklich benannt. Zum anderen lassen sich die jeweiligen Vollzugsbehörden als die nach der innerstaatlichen Rechtsordnung zuständigen Hoheitsträger der insgesamt verpflichteten Vertragspartei „Bundesrepublik“ als Verpflichtete benennen. Eine Notwendigkeit weiterer gesetzlicher Konkretisierung ist in Bezug auf die Adressaten der Bestimmung nicht gegeben.

Unabhängig von der - sogleich zu klärenden Frage - ob die Bestimmung ein bloßes Auskunftsrecht enthält oder auch einen Akteneinsichtsanspruch geschaffen hat, ist diese auch insofern ohne weitere Rechtssetzungsakte vollziehbar, als sie den Gegenstand des Anspruchs klar erkennen lässt: Es geht um solche Informationen, die der Ausschuss benötigt, um seinem Kontrollauftrag nachkommen zu können. Dies umfasst alle Informationen über die Institutionen des Freiheitsentzuges als auch generell über die Art und Weise der Durchführung des Freiheitsentzuges, selbstverständlich aber auch alle Informationen in Bezug auf die dem Freiheitsentzug unterworfenen Personen. Innerhalb dieses Spektrums muss dem Ausschuss das Auswahlermessen zustehen, so dass er selbst bestimmt, welche Informationen ihm mitzuteilen sind. Dem Begehren des Ausschusses könnte dann nur entgegen gehalten werden, dass die angeforderten Informationen in keinem Zusammenhang mit seinem Kontrollauftrag stehen.

Festzuhalten bleibt daher zunächst, dass Art. 8 Abs. 2 lit.d CPT in beiden Auslegungsvarianten (Auskunft/Akteneinsicht) eine unmittelbar vollziehbare, self-executing Norm ist.

\section{(3) Auskunft oder Akteneinsicht - oder Auskunft und Akteneinsicht?}

Schließlich kommt es darauf an, ob es sich bei der Vertragsbestimmung lediglich um ein Recht auf bloße Auskunft handelt oder ob diese auch das Recht auf Einsicht in die Akten umfasst. Die gegenüber dem CPT bislang vertretene restriktive Rechtsauffassung geht von einem bloßen Auskunftsrecht aus und hat bei der Auslegung anscheinend ausgehend von der deutschen Vertragsübersetzung - die Unterscheidung zwischen Auskunfts- bzw. Akteneinsichtsanspruch der Gefangenen im deutschen Vollzugsrecht vor Augen: ${ }^{80}$ So unterscheidet $\S 185$ StVollzG zwischen dem umfassenden Auskunfts-

80 So auch SBJL-Schmid (Fn 35), § 180 Rn 34, wenn sie dem CPT gem. Art. 8 Abs. 2 lit. d CPT lediglich ein Auskunftsrecht zugesteht. 
anspruch „nach Maßgabe des $\S 19$ des Bundesdatenschutzgesetzes“ und dem Recht auf Einsicht in die Akten, das nur dann greift, wenn zur Wahrung eines bestimmten rechtlichen Interesses die bloße Auskunftsgewährung nicht ausreichend und der Betroffene deshalb auf die Einsicht in die Akten angewiesen ist. ${ }^{81}$ Aus dieser Beschränkung des Akteneinsichtsrechtes im deutschen Vollzugsrecht wird offenbar die Schlussfolgerung gezogen, dass auch die Europäische Anti-Folter-Konvention bzw. das deutsche Vertragsgesetz in Art. 8 Abs. 2 lit. d nur ein Auskunftsrecht ohne Recht auf Akteneinsicht meinen kann.

Dies ist jedoch fraglich.

Selbst wenn man - der strengen Transformationstheorie folgend - sich bei der Auslegung ausschließlich an nationalrechtlichen Auslegungskriterien orientiert, kann die Gewährung von Akteneinsicht rechtssystematisch als ein Unterfall der Auskunftsgewährung eingeordnet werden: Denn der vollzugliche Auskunftsanspruch kann nach dem Ermessen der Anstalt auch durch die Gewährung von Akteneinsicht erfüllt werden. ${ }^{82}$ Festhalten lässt sich somit, dass zwar $§ 185$ StVollzG das Recht des Gefangenen auf Akteneinsicht von besonderen, einschränkenden Voraussetzungen abhängig macht, dass aber nicht die Akteneinsicht außerhalb des Begriffes und des Regelungsbereichs der Auskunftsgewährung liegt. Wird dem CPT somit ein Auskunftsanspruch zuerkannt, so schließt das nicht die Gewährung von Akteneinsicht aus sondern räumt den Vollzugsbehörden lediglich ein Ermessen darüber ein, wie sie den Anspruch erfüllen will.

Allerdings spricht gegen eine strikte Zugrundelegung der - vermeintlichen - deutschen vollzuglichen Begriffssystematik für die Interpretation der Konvention bereits der Zeitpunkt der Verabschiedung und des Inkrafttretens der Konvention und des deutschen Vertragsgesetzes, die fast ein Jahrzehnt vor der Einführung der Datenschutzbestimmungen in das StVollzG im Jahr $1998^{83}$ liegen.

Die restriktive Orientierung an dem innerstaatlichen vollzuglichen Auskunftsrecht verliert zusätzlich an Gewicht, wenn man sich den völkerrechtlichen Entstehungshintergrund vergegenwärtigt und den maßgeblichen englischen Originaltext der Bestimmung betrachtet, der einen verbindlicheren Touch als die deutsche Übersetzung hat. Die dort verwendete Formulierung lautet: „....provide the Committee with the following facilities [..]: [..] d. other information available to the Party [..]." Es kann schon fraglich sein, ob die Übersetzung von „facilities“ mit „Erleichterung“ so glücklich ist. Die Formulierung ,to provide with information“ lässt sich vielleicht treffender mit „mit Infor-

81 Im wesentliche gleichlautende Auskunftsrechte finden sich in Art. 203 BayStVollzG, § 126 HmbStVollzG, § 64 HessStVollzG und § 198 NJVollzG; restriktiver: § 49 JVollzGB I BW, vgl. hierzu SBJL-Schmid (Fn 35), § 185 Rn 28.

82 AK-Goerdeler/Weichert (Fn 35), § 185 Rn 3; vgl. für das Landes-Datenschutzrecht auch Unabhängiges Landeszentrum für Datenschutz Schleswig-Holstein (Hrsg), Datenschutz leicht gemacht, Tipps und Hinweise zur Anwendung des Landesdatenschutzgesetzes, Kiel 2002 (https://www.datenschutzzentrum.de/download/hinwldsg.pdf [8.11.2012]), § 27 Rn 12.

83 im Rahmen des Vierten Strafvollzugsänderungsgesetzes vom 26.8.1998, BGB1.I 1998, S. 2461. Zur Entstehungsgeschichte des vollzuglichen Datenschutzes: AK-Goerdeler/Weichert (Fn 35), vor $§ 179 \mathrm{Rz} 1 \mathrm{ff}$. 
mationen zu versorgen“ oder „Informationen zugänglich zu machen“ übersetzen. ${ }^{84}$ Dann käme der Gedanke, es könnte sich lediglich um ein Recht auf bloße Auskunft ohne Akteneinsicht handeln, erst gar nicht auf.

Schließlich ergibt sich ein anderes Bild, wenn - wie vom Bundesverfassungsgericht gefordert $^{85}$ - der völkerrechtliche Entstehungszusammenhang berücksichtigt und die entsprechende Vertragsnorm auch nach völkerrechtlichen Gesichtspunkten ausgelegt wird. Nationales Recht ist demnach unter Berücksichtigung völkerrechtlicher Verträge so auszulegen, dass ein Verstoß gegen Völkerrecht möglichst vermieden wird. ${ }^{86} \mathrm{Nach}$ Art. 31 Abs. 1 WVK ist ein Vertrag, ,nach Treu und Glauben in Übereinstimmung mit der gewöhnlichen, seinen Bestimmungen in ihrem Zusammenhang zukommenden Bedeutung und im Lichte seines Zieles und Zweckes auszulegen.“

Ziel der Konvention ist die Verhinderung von Folter und erniedrigender und unmenschlicher Behandlung oder Strafe durch die Etablierung eines präventiv ausgerichteten Kontrollmechanismus: Behandlungen von Gefangenen, die nicht den menschenrechtlichen Standards entsprechen, sollen dadurch verhindert werden, dass der Ausschuss einen unmittelbaren Zugang zu Orten der Freiheitsentziehung hat und so die dort herrschenden Zustände selbst und unmittelbar wahrnehmen kann. Diese Wahrnehmungen bewertet der Ausschuss, macht sie zum Gegenstand seines Berichtes und spricht Empfehlungen über die Behebung etwaiger Missstände aus. Es entspricht dieser Herangehensweise, dass der Ausschuss nicht nur die Orte der Freiheitsentziehung, sondern auch die Unterlagen über die Freiheitsentziehung selbst, unmittelbar und unabhängig von weiteren Voraussetzungen in Augenschein nehmen kann. Im Umkehrschluss entwertet es diesen Ansatz wesentlich, wenn dem Ausschuss dieses Recht nicht zugestanden wird.

Mit dieser Ausrichtung der Konvention ist es nicht zu vereinbaren, die Bestimmung des Art. 8 Abs. 2 S. 1 lit. d CPT so zu verstehen, dass er dem Ausschuss nur Anspruch auf eine Auskunft durch die verantwortlichen Behörden gewähren will, so dass er sich mit indirekten, von den verantwortlichen Stellen zusammengestellten und formulierten Informationen zufrieden geben müsse. Eine Auslegung, die ein Recht auf Akteneinsicht in den Anspruch des Ausschusses nach Art. 8 Abs. 2 lit. d CPT einbezieht, ist somit nicht nur möglich, sondern geboten.

84 Dieselben Übersetzungsprobleme finden sich auch in der deutschen Fassung des „Explanatory Report" zur Konvention, wobei dort die sprachlichen Unebenheiten noch deutlicher zutage treten, etwa wenn es die Passage „Sub-paragraph (d) obliges Parties to provide the Committee with information available to them which is necessary for the Committee to carry out its task. Access to information will clearly be of great importance to the Committee." dort damit übersetz wird, Unterabsatz (d) verpflichte die Vertragsparteien, dem Ausschuss die „,verfügbaren Auskünfte zu liefern“ und dass „,der Zugang zu Auskünften“ für den Ausschuss von großer Bedeutung sei, European Committee for the Prevention of Torture and Inhuman or Degrading Treatment or Punishment (CPT): European Convention for the Prevention of Torture and Inhuman or Degrading Treatment or Punishment, Text of the Convention and Explanatory Report, CPT/Inf/C (2002) 1, Strasbourg 2002 (http://www.cpt.coe.int/en/documents/eng-convention.pdf [30.10.2012]), AbsNr. 64. Deutsche Fassung: Text der Konvention und des Erläuternden Berichts, CPT/Inf/C (2002) 2, Straßburg 2002 (www.cpt.coe.int/lang/ deu/deu-convention.pdf [30.10.2012]).

85 BVerfGE 111, 307, $317 \mathrm{f}$.

86 BVerfGE 111, 307, $317 \mathrm{f}$. 


\section{(4) Vorbehalt des innerstaatlichen und des Standesrechts}

Nach Satz 2 des Art. 8 Abs. 2 lit. d 2 CPT hat der Ausschuss im Rahmen seines Informationsrechtes das innerstaatliche Recht und das Standesrecht zu beachten. Schließt dieser Vorbehalt die unmittelbare Einsichtnahme in Gefangenenpersonalakten und insbesondere in die Krankenakten aus?

Erkennbar ist dieser Vorbehalt nicht so zu verstehen, dass das Informationsrecht des Ausschusses von der gesonderten innerstaatlichen Einräumung eines Informationsrechtes abhängen und nur in dessen Grenzen bestehen soll. Dagegen, dass die Konvention den Informationsanspruch soweit zurücknehmen will, spricht zunächst die Formulierung des Vorbehalts: Nach der deutschen Übersetzung sind innerstaatliche Rechtsvorschriften und das Standesrecht „,bei der Beschaffung solcher Auskünfte“ (,, In seeking such information... ") vom Ausschuss zu beachten. Diese Formulierung spricht dafür, dass sich der Vorbehalt auf die Modalitäten der Informationsgewährung bezieht - bspw. auf Regelungen zur Zuständigkeit, zum Verfahren, zur Abwicklung und auf den Umgang mit den personenbezogenen Daten von Dritten. Der Wille, unmittelbare Verpflichtungen und Befugnisse zu begründen, steht durch den Vorbehalt nicht in Frage und zeigt sich auch in anderen Bestimmungen, die die Vertragsparteien verpflichten, bspw. in dem Recht auf unmittelbaren Zugang zu den Orten der Freiheitsentziehung. Auch die Erläuterungen des Explanatory Report stellen die unmittelbare Verpflichtung der Vertragsparteien zur Informationsgewährung nicht in Frage: So wird dort ausgeführt, dass die Bestimmung die Vertragsparteien verpflichte, dem Ausschuss die erforderlichen und verfügbaren Auskünfte zu liefern, es werde aber anerkannt, „dass in den Mitgliedstaaten besondere Regeln für die Offenlegung von Auskünften gelten können“. 87

Dennoch könnten sich Beschränkungen insbesondere für den Zugang zu Krankenakten ergeben. Medizinische Daten haben eine besondere Sensibilität für den Betroffenen. Zugleich gehört die Schweigepflicht traditionell zu den von der Rechtsordnung anerkannten ${ }^{88}$ Berufspflichten der Ärzte. Für den Kontrollauftrag der Präventionsinstitutionen sind die Gesundheitsakten aber von besonderer Bedeutung, weil sich in diesen Aufzeichnungen Anhaltspunkte über die Spuren von Übergriffen, über die gesundheitlichen Folgen der Inhaftierung und über Art und Qualität der gesundheitlichen Versorgung finden können. Der Gesundheitsversorgung kommt in einer Situation, in der sich der Gefangene in unmittelbarer und umfassender staatlicher Verantwortung befindet, eine zentrale Bedeutung in der Haftgestaltung zu. Vor diesem Hintergrund ist klärungsbedürftig, ob das von der Konvention konstituierte Informationsrecht auch Krankenakten der Gefangenen umfasst.

Für eine Beschränkung des Informationsanspruchs und eine besondere Behandlung der medizinischen Daten gibt es drei Anknüpfungspunkte:

1. Der allgemeine Subsidiaritätsvorbehalt des BDSG: Nach $§ 1$ Abs. 3 S. 2 BDSG bleibt u.a. die Pflicht zur Wahrung von Berufsgeheimnissen, die nicht auf gesetzli-

87 CPT, Explanatory Report (Fn 84), AbsNr. 64.

88 bspw. im Rahmen des strafrechtlichen Schutzes von Geheimnissen nach $\S 230$ StGB, in der Anerkennung eines beruflichen Zeugnisverweigerungsrechtes gemäß $§ 52$ StPO oder in der - allerdings ziemlich löchrigen - Verschwiegenheitspflicht der Anstaltsärzte gegenüber der Anstaltsleitung in $\S 182$ Abs. 2 StVollzG. 
chen Vorschriften beruhen, von den Regelungen des BDSG unberührt. Gemeint sind damit die auf Standesrecht beruhenden Geheimhaltungspflichten, bspw. der Ärzte. Weitergehende Geheimhaltungspflichten werden demnach durch die Regelungen des BDSG nicht nivelliert. ${ }^{89}$ Dieser Vorbehalt gilt auch für die Wahrnehmung der Kontrollrechte nach $\S 14$ Abs. 3 BDSG, auf die $\S 180$ Abs. 3 StVollzG Bezug nimmt. ${ }^{90}$

2. Nach $\S 180$ Abs. 10 StVollzG hat eine Übermittlung personenbezogener Daten u.a. dann zu unterbleiben, wenn die für Berufsgeheimnisträger im Vollzug geltenden besonderen Verschwiegenheitsverpflichtungen und -rechte nach $\S 182$ Abs. 2 StVollzG entgegenstehen..$^{91}$ Mit dieser Einschränkung der Übermittlungsbefugnis wird ebenfalls u.a. auf die berufsständische Verschwiegenheitsverpflichtung der Ärzte Bezug genommen, die nach $\S 182$ Abs. 2 StVollzG auch gegenüber der Anstaltsleitung und der Aufsichtsbehörde ${ }^{92}$ zur Verschwiegenheit verpflichtet und berechtigt sind. Abgesehen von den weiter bezeichneten Informationspflichten bzw. -befugnissen gegenüber der Anstaltsleitung regelt $\S 182$ Abs. 2 S. 4 StVollzG, dass sonstige Offenbarungsbefugnisse unberührt bleiben.

3. Der schon angesprochene Vorbehalt des Art. 8 Abs. 2 lit. d CPT bezieht sich für die „Beschaffung von Auskünften“ ausdrücklich auch auf das Standesrecht und damit auf die ärztliche Verschwiegenheitspflicht.

Diese Ansatzpunkte laufen somit jeweils darauf hinaus, dass sich die Übermittlungsbefugnisse und Informationsrechte an Inhalt und Reichweite der berufsständischen ärztlichen Schweigepflicht ausrichten.

Die zentrale Bedeutung des Schweigegebots für den ärztlichen Berufsstand lässt sich schon daran bemessen, dass die Verschwiegenheit im hippokratischen Eid, den jeder Arzt abzulegen hat, bevor er den Beruf ausüben darf, ausdrücklich angesprochen wird..$^{93}$ Inhalt und Umfang der ärztlichen Schweigepflicht lassen sich aus der MusterBerufsordnung (MBO-Ä) für die in Deutschland tätigen Ärztinnen und Ärzte ${ }^{94}$ sowie aus den Empfehlungen der Bundesärztekammer und der Kassenärztlichen Bundesvereinigung zur ärztlichen Schweigepflicht ${ }^{95}$ bestimmen. $\S 9$ Abs. 1 MBO-Ä verpflichtet die Ärzte zunächst dazu, über das ihnen in ihrer Eigenschaft als Arzt Anvertraute oder Bekanntgewordene zu schweigen. Zu den Offenbarungsbefugnissen heißt es in Abs. 2:

89 Gola/Schomerus (Fn 44), § 1 Rn 25. DKWW-Weichert (Fn 44), § 1 Rn 14.

$90 \mathrm{Gola} / \mathrm{Schomerus} \mathrm{(Fn} \mathrm{44),} \mathrm{§} 14 \mathrm{Rn} 24$.

91 SBJL-Schmid (Fn 35), § 180 Rn 23 \& 49; a.A.: Arloth, Strafvollzugsgesetze, Kommentar, 3. Auflage, München 2011, 180 Rn 5; Weichert in: Feest, Johannes (Hrsg), Strafvollzugsgesetz, Kommentar, 5. Auflage, Neuwied 2006 (5. Auflage), § 180 Rn 27.

92 AK-Goerdeler/Weichert (Fn 35), § 182 Rn 33.

93 „Ich werde alle mir anvertrauten Geheimnisse auch über den Tod der Patientin oder des Patienten hinaus wahren.“, abgedruckt in der (Muster-)Berufsordnung für die in Deutschland tätigen Ärztinnen und Ärzte (Fn 94), S. 4.

94 (Muster-)Berufsordnung für die in Deutschland tätigen Ärztinnen und Ärzte - MBO-Ä 1997 - in der Fassung der Beschlüsse des 114. Deutschen Ärztetages 2011 in Kiel (http://www.bundesaerztekammer.de/downloads/mbo_08_20111.pdf [30.10.2012]).

95 Bundesärztekammer \& Kassenärztliche Bundesvereinigung: Empfehlungen zur ärztlichen Schweigepflicht, Datenschutz und Datenverarbeitung in der Arztpraxis, Deutsches Ärzteblatt 13/2008, S. 1026-1030 (http://www.bundesaerztekammer.de/downloads/Empfehlung_Schw eigepflicht_Datenschutz.pdf [30.10.2012]). 
„,(2) Ärztinnen und Ärzte sind zur Offenbarung befugt, soweit sie von der Schweigepflicht entbunden worden sind oder soweit die Offenbarung zum Schutze eines höherwertigen Rechtsgutes erforderlich ist. Gesetzliche Aussage- und Anzeigepflichten bleiben unberührt. Soweit gesetzliche Vorschriften die Schweigepflicht der Ärztin oder des Arztes einschränken, soll die Ärztin oder der Arzt die Patientin oder den Patienten darüber unterrichten."

Eine Offenbarungs- oder Übermittlungsbefugnis kann sich damit sowohl aus einer Einwilligung des Patienten, aus dem Schutz höherwertiger Rechtsgüter als auch aus gesetzlichen Einschränkungen der Schweigepflicht ergeben.

Auch die Empfehlungen zur ärztlichen Schweigepflicht der Bundesärztekammer und der Kassenärztlichen Bundesvereinigung stellen fest, dass ,eine Durchbrechung der ärztlichen Schweigepflicht [..] zulässig [ist], wenn gesetzliche Vorschriften dem Arzt eine Pflicht oder ein Recht zur Offenbarung auferlegen bzw. geben“96 und dass „die Übermittlung von Patientendaten [..] nur zulässig [ist], wenn sie entweder durch eine gesetzliche Vorschrift, durch die Einwilligung des Patienten oder aber durch einen besonderen Rechtfertigungsgrund legitimiert ist". 97

Zunächst zeigt sich, dass die Wahrung der Vertraulichkeit für die ärztliche Berufsausübung nach wie vor einen besonders hohen Stellenwert hat und von der Ärzteschaft selbst als typische, ja geradezu fundamentale Verbürgung ihrer Berufstätigkeit gesehen wird. Andererseits hat die standesrechtliche Ausformung der Schweigepflicht die komplexer werdende, von zunehmender Arbeitsteilung und Verschränkungen mit anderen Institutionen (Krankenhäusern, Krankenkassen, Sozialleistungsträgern, Abrechnungsstellen usw.) geprägte Arbeitswelt des Gesundheitswesens zu berücksichtigen, die teilweise ohne eine Weitergabe von Patientendaten gar nicht funktionieren könnte. So ist der Arzt einerseits zur Verschwiegenheit verpflichtet, andererseits gesteht ihm die standesrechtliche Ausgestaltung des Schweigerechts die Übermittlung von Patientendaten aufgrund gesetzlicher Übermittlungsbefugnisse durchaus zu. Soweit nun ihrerseits Übermittlungsbefugnisse unter dem Vorbehalt der standesrechtlichen Schweigepflicht stehen, wird dadurch zumindest zum Ausdruck gebracht, dass die Übermittlung nicht der ärztlichen Ethik und der Orientierung am Patienten zuwiderlaufen darf und dass sie im Zusammenhang mit der ärztlichen Behandlungstätigkeit stehen muss. Im Übrigen ist durch Auslegung und unter Abwägung der berührten Interessen zu ermitteln, ob eine Übermittlung von Patientendaten zulässig ist oder nicht.

Für eine Zulässigkeit der Übermittlung sprechen im hiesigen Kontext folgende Überlegungen:

Zunächst besteht eine gewisse Kongruenz in den Zielen, die das mit der Europäischen Anti-Folter-Konvention etablierte Kontrollsystem verfolgt, und dem am Wohl des Patienten orientierten Behandlungsauftrag der Ärzte. ${ }^{98}$ Die Kontrollen durch das CPT

96 Bundesärztekammer \& Kassenärztliche Bundesvereinigung (Fn ), S.1026.

97 Bundesärztekammer \& Kassenärztliche Bundesvereinigung (Fn ), S.1029.

98 vgl. § 2 Abs. 2 MBO-Ä (Fn 94): „Ärztinnen und Ärzte haben ihren Beruf gewissenhaft auszuüben und dem ihnen bei ihrer Berufsausübung entgegengebrachten Vertrauen zu entsprechen. Sie haben dabei ihr ärztliches Handeln am Wohl der Patientinnen und Patienten auszurichten. Insbesondere dürfen sie nicht das Interesse Dritter über das Wohl der Patientinnen und Patienten stellen.“. 
dienen dazu, Gefangene präventiv vor Übergriffen durch Mitgefangene oder Bedienstete sowie vor gesundheitsbeeinträchtigenden Haftbedingungen zu schützen. Somit geht es auch um die körperliche und seelische Gesunderhaltung des Betroffenen.

Die Kontrollen sind auch im objektiven Interesse der Gefangenen. Nachteile sind mit den Kontrollen nicht verbunden, ein entgegenstehendes Interesse, außer allgemein dem, dass körperliche oder seelische Beschwerden Dritten nicht bekannt werden sollen, ist nicht ersichtlich. Die Bedeutung dieses Interesses mag sich nicht ganz nivellieren, verringert sich aber erheblich dadurch, dass die Mitglieder des CPT selbst zur strikten Verschwiegenheit verpflichtet sind. ${ }^{99}$

$\mathrm{Zu}$ berücksichtigen ist auch, dass die alternative Gestaltung des Verfahrens zur Einsichtnahme in Krankenakten, nämlich über die Einholung einer Einwilligung des Betroffenen, langwieriger und störungsanfällig ist, bspw. weil Gefangene aus Angst vor Repressionen mit der Erteilung der Einwilligung zögern oder diese nicht erteilen. ${ }^{100}$ Außerdem kann ein solches Verfahren nicht in demselben Maße potentielle Manipulationen durch die Einrichtungen ausschließen, die durch die Vor-Ort-Besuche kontrolliert werden sollen.

All dies spricht dafür, das ärztliche Schweigerecht und die auf dieses verweisenden Vorbehalte in $\S 180$ Abs. 10 StVollzG, $\S 1$ Abs. 3 S.2 BDSG und Art. 8 Abs. 2 lit.d S. 2 CPT nicht so zu verstehen, dass sie einer Einsichtnahme des CPT in die vollzuglichen Krankenakten grundsätzlich entgegenstehen. Allerdings kann und muss dieser Vorbehalt so interpretiert werden, dass er das Komitee zu einem besonders sensiblen Umgang mit den Informationen verpflichtet, die es aus der Einsichtnahme in ärztliche Unterlagen gewonnen hat. Bspw. erfolgen Äußerungen der Gefangenen gegenüber dem Arzt unter Vertrauen auf die ärztliche Verschwiegenheit; das CPT wird daher so vorgehen müssen, dass dem Personal der Anstalt und der Aufsichtsbehörde die von Gefangenen gemachten Äußerungen nicht ohne deren Einwilligung bekannt werden.

\section{(5) Ergebnis}

Mit diesen Maßgaben kann $\S 180$ Abs. 3 StVollzG iVm § 14 Abs. 3 BDSG iVm Art. 8 Abs. 2 lit. d CPT-VertrG als ausreichende Rechtsgrundlage angesehen werden, um dem CPT die Einsichtnahme in vollzugliche Gefangenen- und Krankenakten zur Ausübung seiner Kontrollbefugnisse zu ermöglichen.

\section{c) Akteneinsichtsrecht aufgrund OPCAT}

Das OPCAT sieht - wie bereits dargestellt - zwei Mechanismen der Folter-Prävention vor, nämlich einerseits die Einrichtung des Vereinte Nationen-Unterausschusses zur Verhütung von Folter und seine Ausstattung mit Kontrollbefugnissen und andererseits

99 Art. 11 CPT: „(1) Die Informationen, die der Ausschuss bei seinem Besuch erhält [..] sind vertraulich. [..] (3) Personenbezogene Daten dürfen jedoch nicht ohne ausdrückliche $\mathrm{Zu}-$ stimmung des Betroffenen veröffentlicht werden".

100 Darauf weist auch das CPT hin, insbesondere bei Gefangenen, die Opfer von Übergriffen geworden sind, CPT, Bericht (Fn 3), AbsNr. 7. 
die Etablierung eines nationalen Präventionsmechanismus. Beiden sollen nach dem OPCAT u.a. die eingangs genannten Befugnisse zustehen, nämlich Orte der Freiheitsentziehung aufzusuchen und Zugang zu allen für ihren Kontrollauftrag relevanten Informationen $\mathrm{zu}$ erhalten. ${ }^{101}$

\section{(1) Gesetzgebungskompetenz für den Strafvollzug}

Anders als bei der Europäischen Antifolterkonvention sieht es hinsichtlich der Gesetzgebungskompetenz des Bundes beim OPCAT aus: Das Vertragsgesetz ist im Jahr 2008 verabschiedet worden und in Kraft getreten ${ }^{102}$ - also nach der Föderalismusreform, durch die der Bereich ,Strafvollzug“ aus der Gesetzgebungskompetenz des Bundes nach Art. 74 Abs. 1 Nr. 1 GG herausgenommen wurde. ${ }^{103}$ Die Länder haben seither die ausschließliche Gesetzgebungskompetenz für den Kompetenztitel „Strafvollzug“. Der Einführung des internationalen und des nationalen Präventionsmechanismus durch das OPCAT-VertrG wird man zudem eine grundsätzliche Bedeutung nicht absprechen können, die über eine bloße Anpassung einzelner Vorschriften des StVollzG hinaus geht daher kommt letztlich auch keine Bundeskompetenz infolge einer bloßen Anpassung des nach Art. 125 a Abs. 1 GG fortgeltenden Bundesrechtes in Betracht. ${ }^{104}$ Der Bund konnte zum Zeitpunkt der Verabschiedung und des Inkrafttretens des Vertragsgesetzes zum OPCAT zwar über andere Arten der Freiheitsentziehung Regelungen treffen, für die er Kompetenztitel besaß (bspw. die Abschiebungshaft), nicht aber für den Bereich „Strafvollzug“.

Wie bei „normalen“ Bundesgesetzen, die unter einer (teilweisen) Überschreitung der Gesetzgebungskompetenz des Bundes zustande gekommen sind, führt dieser Kompetenzverstoß jedoch nicht von selbst zur Unwirksamkeit oder Unanwendbarkeit des Ge-

101 Hof/Mohsen, Folterprävention in Deutschland, Eine erste Bilanz der Arbeit der Nationalen Stelle zur Verhütung von Folter, MschrKrim 2012, 296.

102 Das Vertragsgesetz trat laut seinem Art. 3 am Tag nach der Verkündung, die durch das BGB1.II v. 2. September 2008, S.854, erfolgte, in Kraft, somit am 3. September 2008. Das Abkommen trat gemäß seinem Art. 28 für die Bundesrepublik am 3. Januar 2009 in Kraft.

103 Gesetz zur Änderung des Grundgesetzes (Artikel 22, 23, 33, 52, 72, 73, 74, 74 a, 75, 84, 85, $87 \mathrm{c}, 91 \mathrm{a}, 91 \mathrm{~b}, 93,98,104 \mathrm{a}, 104 \mathrm{~b}, 105,107,109,125 \mathrm{a}, 125 \mathrm{~b}, 125 \mathrm{c}, 143 \mathrm{c})$ vom 28. August 2006, BGB1.I 2006, S.2034.

104 SBHH-Maiwald (Fn 52), Art. 125 a GG Rn 6; vgl. BVerfGE 111, 1, 10 zu Art. 125 a Abs. 2 GG. 
setzes: ${ }^{105}$ Es gilt auch hier das Verwerfungsmonopol des Bundesverfassungsgerichtes. Soweit das OPCAT-Vertragsgesetz Vertragsnormen enthält, die self-executing sind, sind diese somit unmittelbar anwendbares Recht - solange bis das Bundesverfassungsgericht sie im Rahmen eines Normenkontrollverfahrens in ihrer Geltung einschränkt oder gar für nichtig erklärt. Sollten die Länder hiermit nicht einverstanden sein, wäre es an ihnen, im Rahmen eines Bund-Länder-Streites das Bundesverfassungsgericht anzurufen.

\section{(2) Self-Executing}

Hinsichtlich der Vertragsnormen, die den nationalen Präventionsmechanismus betreffen, fehlt es offenkundig an einer unmittelbaren Umsetzbarkeit: Der nationale Mechanismus wird durch das OPCAT bzw. dem dazugehörigen VertrG nicht geschaffen, vielmehr enthält das Fakultativprotokoll lediglich die Verpflichtung, diesen Mechanismus einzurichten. Hierfür sind weitere konkretisierende Rechtsnormen erforderlich, die u.a. Status und Struktur der einzurichtenden Stelle und ihre Besetzung regeln müssen.

Die Länder sind dem Auftrag zur Einrichtung eines nationalen Präventionsmechanismus dadurch nachgekommen, dass sie mit dem „Staatsvertrag über die Einrichtung eines nationalen Mechanismus aller Länder nach Artikel 3 des Fakultativprotokolls vom 18. Dezember 2002 zu dem Übereinkommen der Vereinten Nationen gegen Folter und andere grausame, unmenschliche oder erniedrigende Behandlung oder Strafe" 106 (StaatsV) vom 1. Dezember 2009 die Errichtung einer Kommission zur Verhütung von Folter vereinbart haben. Diese Länderkommission bildet demnach zusammen mit der Bundesstelle zur Verhütung von Folter ${ }^{107}$ die Nationale Stelle zur Verhütung von Folter und hat ihre Arbeit am 24. September 2010 in Wiesbaden aufgenommen. ${ }^{108}$ Für den Abschluss des StaatsV und seine Eingliederung in das jeweilige Landesrecht hatten die Länder nach den obigen Ausführungen auch gem. Art. 70 Abs. 1 GG die erforderliche Gesetzgebungskompetenz.

105 Allerdings wäre in dem hier zu behandelnden spezialgelagerten Sonderfalls (J. Jonas) eines unter teilweiser Kompetenzwidrigkeit zustande gekommenen Vertragsgesetzes auch die Position vertretbar, dass das Gesetz für die Bereiche, in denen der Bund keine Gesetzgebungskompetenz hat, lediglich die Mitwirkung der Legislative im Rahmen der Ratifizierung sicherstellt, aber kein unmittelbar anwendbares innerstaatliches Recht schafft. Hierfür spräche die Erforderlichkeit des Vertragsgesetzes im Rahmen des Ratifizierungsverfahrens, so dass auch das BVerfG das Gesetz nicht als nichtig klassifizieren, sondern lediglich seinen Anwendungsbereich einschränken dürfte. Außerdem hat der Bundesgesetzgeber keine inhaltliche Ausgestaltungsmöglichkeit, er kann nur den vorgegebenen Vertragstext ,absegnen“. Würde dieser Auffassung gefolgt, würde gegenwärtig eine innerstaatliche Rechtsgrundlage für die Informationsansprüche des VN-Unterausschusses fehlen, und die Länder wären völkerrechtlich und aus Bundestreue verpflichtet, entsprechende Umsetzungsgesetze zu schaffen.

106 etwa in Baden-Württemberg: BW GBl. v. 7.12.2009, S. 681; in Niedersachsen: Nds.GVB1. 2010 S. 54; in Sachsen: Sächsisches GVB1 2010, S. 146.

107 S. hierzu den Organisationserlass des Bundesministeriums der Justiz vom 20. November 2008; zu finden über www.antifolterstelle.de/rechtsgrundlagen [15.10.2012].

108 www.antifolterstelle.de/rechtsgrundlagen [15.10.2012]. 
Art. 1 StaatsV bestimmt, dass die vertragsschließenden Länder gemeinsam eine Kommission zur Verhütung von Folter einrichten. Die näheren Einzelheiten über Mitglieder, Sekretariat, Sitz, Arbeitsweise, Zusammenarbeit mit der Bundesstelle und die Finanzierung werden in den Artikel 4 bis 9 geregelt. Über die Aufgaben und Befugnisse der Kommission bestimmt Artikel 2:

(1) Die Kommission hat die Aufgabe, zur Verhütung von Folter Orte der Freiheitsentziehung im Sinne des Artikels 4 des Fakultativprotokolls im Zuständigkeitsbereich der Länder aufzusuchen, auf Missstände aufmerksam zu machen und gegebenenfalls Verbesserungsvorschläge zu machen.

(2) Den Mitgliedern der Kommission stehen einzeln oder gemeinsam die in Artikel 19 des Fakultativprotokolls genannten Befugnisse zu. Die Länder gewähren ihnen die in Artikel 20 des Fakultativprotokolls genannte Rechte und Befugnisse. [..]

Der Staatsvertrag ist in jedem Bundesland durch Vertragsgesetz in Kraft gesetzt worden, ${ }^{109}$ so dass nun in jedem Land ein der innerstaatlichen Kompetenzordnung entsprechender Rechtsakt vorliegt.

Durch diesen Rechtsakt werden der Nationalen Stelle auch für jedes Bundesland unmittelbar anwendbare Kontrollbefugnisse zugeordnet, denn Art. 2 Abs. 2 StaatsV weist der Länderkommission die in den Art. 19 und 20 OPCAT vorgesehenen Rechte zu auch die Informationsrechte gemäß Art. 20 lit. a \& b OPCAT. ${ }^{110}$ Hinsichtlich dieser Informationsrechte ist nun die Länderkommission als Rechte-Inhaberin konstituiert worden.

Des Weiteren ergeben sich auch die Verpflichtungen hinreichend deutlich aus dem Kontext des Vertrages: wie auch beim CPT sind hier diejenigen Hoheitsträger gemeint, die die Kontrolle über die Orte der Freiheitsentziehungen ausüben, also die Vollzugsanstalten und ihre Aufsichtsbehörden. Auch der Gegenstand der Verpflichtung ist nicht unbestimmter als bei anderen inländischen Bestimmungen üblich: Die Verpflichtung besteht für die betreffenden Behörden darin, der Nationalen Stelle die bei ihr vorhandenen Informationen zugänglich zu machen, wobei es sich bei den Informationen gemäß Art. 20 lit. a OPCAT um allgemeine Informationen über die Einrichtungen handelt, während sich lit. b auch auf Informationen über die individuellen Bedingungen des Freiheitsentzuges bezieht. Die durch Art. 20 lit. a \& b OPCAT iVm Art. 2 StaatsV verliehenen Befugnisse sind somit ausreichend bestimmt, um sie ohne weitere legislative Akte anwenden zu können.

Dasselbe gilt für die Informationsrechte, die dem VN-Unterausschuss durch das OPCAT-VertrG gemäß Art. 14 lit. a \& b zugeordnet werden. Während für den nationalen Präventionsmechanismus unmittelbar anwendbares Recht erst durch den Staatsvertrag geschaffen wird, enthält die OPCAT hinsichtlich des VN-Unterausschusses bereits alle erforderlichen Regelungen. Insofern sind die Vertragsbestimmungen somit auch „self executing".

109 durch das „,Gesetz zu dem Staatsvertrag über die Einrichtung eines nationalen Mechanismus aller Länder nach Artikel 3 des Fakultativprotokolls vom 18. Dezember 2002 zu dem Übereinkommen der Vereinten Nationen gegen Folter und andere grausame, unmenschliche oder erniedrigende Behandlung oder Strafe", etwa in Baden-Württemberg BW GBl. v. 7.12.2009, S.681, in Niedersachsen Nds.GVB1. 2010 S. 54.

110 Hof/Mohsen (Fn 101), MschKrim 2012, 296. 


\section{(3) Auskunft oder Einsicht}

In der Frage einer Beschränkung auf ein bloßes Auskunfts- oder der Einräumung eines Akteneinsichtsrechtes sind die Informationsrechte des OPCAT klarer gefasst als im CPT: Nach Art. 14 Abs. 1 lit. b, 20 lit. b OPCAT sind dem VN-Unterausschuss und der Kommission Zugang zu allen Informationen über die Behandlung der inhaftierten Personen und die Bedingungen der Freiheitsentziehung zu gewähren. Dass Art. 20 lit. b nur einen „Zugang“, aber nicht wie Art. 14 Abs. 1 lit. b einen „unbeschränkten Zugang“ gewähren, bedeutet keine inhaltliche Beschränkung des Informationsrechtes. ${ }^{111}$ Dieser Zugang zu Informationen ist insbesondere durch eine unmittelbare Einsicht in die relevanten Unterlagen herzustellen. ${ }^{112}$ Anhaltspunkte für eine Beschränkung auf ein bloßes Auskunftsrecht ergeben sich aus der Formulierung noch weniger als beim CPT. Wie schon dort wäre es auch bei den vom OPCAT vorgesehenen Institutionen systemwidrig, sollten sie auf einen indirekten und damit gefilterten Zugang zu den relevanten Informationen verwiesen werden.

Nach den obigen Ausführungen gelten die datenschutzrechtlichen Vorbehalte über medizinische Daten ( $§ 180$ Abs. 10 StVollzG, $§ 1$ Abs. 3 S. 2 BDSG) zwar auch für die Informationsrechte aus dem OPCAT, sie führen jedoch auch hier nicht zu einer grundsätzlichen Einschränkung der Informationsrechte des Vereinte Nationen-Unterausschusses oder der Nationalen Stelle.

\section{(4) Ergebnis}

Somit ist festzustellen, dass jeweils durch einen innerstaatlichen Gesetzgebungsakt (CPT-VertrG, OPCAT-VertrG, Vertragsgesetze zum Staatsvertrag der Länder) einer öffentliche Stelle (CPT, Vereinte Nationen-Unterausschuss, Länderkommission als Teil der Nationalen Stelle) unmittelbar anwendbare Kontrollbefugnisse im Sinne des $\S 180$ Abs. 3 StVollzG iVm § 14 Abs. 3 BDSG einschließlich des Rechts auf Einsichtnahme in vollzugliche Unterlagen eingeräumt worden sind. Dieses Recht umfasst auch die unmittelbare Einsichtnahme in die Gefangenenakten, einschließlich der Gesundheitsakten.

\section{2. §180 Abs. 4 S. 2 StVollzG}

Als weitere oder alternative Rechtsgrundlage für ein Akteneinsichtsrecht des CPT, des VN-Unterausschusses und der Nationalen Stelle kommt $§ 180$ Abs. 4 S. 2 StVollzG in Verbindung mit dem CPT-VertrG, dem OPCAT-VertrG bzw. den Landesgesetzen zum Staatsvertrag in Betracht. Abweichend von dem ansonsten abschließenden Charakter der vollzuglichen Datenverwendungsbefugnisse lässt $§ 180$ Abs. 4 S. 2 StVollzG den Rückgriff auf gesetzliche Übermittlungskompetenzen außerhalb des StVollzG zu, wenn

111 so die Begründung zum Entwurf des Vertragsgesetzes der Bundesregierung, BT-Drs. 16/8249, S. 34.

112 so auch Hof/Mohsen (Fn 101), MschrKrim 2012, 296 und 297. 
diese ausdrücklich auf personenbezogene Daten von Gefangenen Bezug nehmen. ${ }^{113}$ Diese Voraussetzung wird durch die Europäische Antifolterkonvention und das Fakultativprotokoll zweifellos erfüllt, beziehen sich die Verträge doch insgesamt auf „Personen, denen die Freiheit entzogen ist" ${ }^{\prime 114}$ um sie vor menschenrechtswidrigen Zuständen in „Orten der Freiheitsentziehung“ zu schützen. Im Übrigen kann auf die obigen Ausführungen über die Schaffung unmittelbar anwendbarer innerstaatlicher Rechtsgrundlagen und ihren jeweiligen Regelungsgehalt verwiesen werden. Damit besteht auch über diesen Weg eine Rechtsgrundlage für eine Akteneinsicht der Präventionsinstitutionen.

\section{Andere Arten des Freiheitsentzuges}

Auf die im Rahmen der Prüfung für den Strafvollzug gewonnene Erkenntnis, dass durch unmittelbar anwendbares innerstaatliches Recht Kontrollinstitutionen mit einem eigenständigen Akteneinsichtsrecht geschaffen worden sind, kann ohne Abstriche auch im Rahmen der Übermittlungsbefugnisse in anderen Vollzugsarten zurückgegriffen werden. Wie dargelegt ist für das jeweilige Rechtsgebiet zu klären, ob das hierfür geltende Datenschutzregime abgeschlossen ist, so dass jeweils bestimmte Verweisungs- oder Öffnungstatbestände gegeben sein müssen, oder ob die genannten Vertragsgesetze für die konkrete Form der Freiheitsentziehung zusammen für die verantwortliche Institution unmittelbar anwendbar sind.

Für andere Arten des Justizvollzuges (Jugendstrafvollzug, Untersuchungshaftvollzug, Sicherungsverwahrungsvollzug, Abschiebungshaft in Justizvollzugseinrichtungen) ist die Rechtslage mit der oben dargestellten identisch: denn entweder sind die Datenschutzvorschriften des StVollzG anzuwenden (bspw. für die Zurückweisungsund Abschiebungshaft aufgrund $\S 422$ Abs. 4 FamFG iVm $\S \S 171$ und 180 StVollzG) oder sie enthalten in dieser Hinsicht inhaltsidentische Datenschutzbestimmungen.

Die Maßregelvollzugsgesetze enthalten in der Regel keine abschließenden Datenschutzbestimmungen - sofern sie überhaupt Regelungen zum Datenschutz enthalten, ${ }^{115}$ sind diese punktuell, ${ }^{116}$ so dass auf allgemeine Datenschutzbestimmungen zurückzugreifen ist. Teilweise finden sich besondere Übermittlungsermächtigungen mit Bezug auf externe Ermächtigungsgrundlagen. ${ }^{117}$

Hinsichtlich einer Unterbringung im Polizeigewahrsam findet sich im Polizeirecht in der Regel eine Übermittlungsbefugnis mit Verweis auf andere gesetzliche Grundla-

113 AK-Goerdeler/Weichert (Fn 35), § 180 Rn 45; ausdrücklich zur CPT: SBJL-Schmid (Fn 35), § 180 Rn 34, wobei diese das Informationsrecht nach Art. 8 Abs. 2 lit. d CPT als bloßes Auskunftsrecht ohne Akteneinsicht versteht.

114 Art. 1 CPT; Art. 1 OPCAT.

115 Keinen Datenschutzteil enthalten bspw. das Hessische Maßregelvollzugsgesetz und das Bayerische Unterbringungsgesetz.

116 Einzelne Datenschutzbestimmung finden sich bspw. im Maßregelvollzugsgesetz NRW (§ 26) und im Maßregelvollzugsgesetz Schleswig-Holstein (§§ $22-24)$.

$117 \S 26$ Abs. 1 MVollzG NRW: „Einzelangaben über persönliche oder sachliche Verhältnisse der Patientinnen und Patienten (Daten) dürfen nur erhoben, gespeichert, genutzt oder übermittelt werden, soweit [..] b) eine andere Rechtsvorschrift dies erlaubt [..]. 
gen, ${ }^{118}$ so dass hierfür unmittelbar auf die Vertragsgesetze zurückgegriffen werden kann.

Bei sozialrechtlichen Unterbringungen (in Jugend- oder Seniorenheimen) ist eine Übermittlung der dabei anfallenden Sozialdaten durch den Träger der öffentlichen Jugendhilfe im Rahmen des Kontrollauftrages der Präventionsmechanismen gem. $\S \S 69$ Abs. 5, 67 c Abs. 3 SGB X zulässig. Bei Unterbringung im Rahmen der Jugendhilfe darf hierdurch der Erfolg der Leistung nicht gefährdet werden (§ 64 Abs. 2 SGB VIII).

\section{Ergebnis}

Die bestehende Rechtslage gibt den Spielraum dafür her, für den Strafvollzug und andere Arten des Freiheitsentzuges von einem Recht des CPT, des Vereinte Nationen-Unterausschusses zur Verhütung von Folter und der Nationalen Stelle zur Verhütung von Folter auf Einsicht in die Gefangenenpersonalakten und die Krankenakten der Gefangenen auszugehen: auch beim CPT ist eine solche Auslegung möglich, wenn nicht gar geboten. Hinsichtlich der Länderkommission der Nationalen Stelle ist durch den Staatsvertrag der Länder die dahingehende Verpflichtung aus dem OPCAT zweifelsfrei umgesetzt worden. Beim Vereinte Nationen-Unterausschuss dürften keine Zweifel darüber bestehen, dass das OPCAT ein Akteneinsichtsrecht vorsieht; hier liegt es in den Händen der Länder, dieses wie im OPCAT-VertrG vorgesehen zu akzeptieren - oder das Bundesverfassungsgericht anzurufen.

Wird jedoch davon ausgegangen, dass ein innerstaatliches Akteneinsichtsrecht für das CPT oder den Vereinte Nationen-Unterausschuss nicht bestehen sollte, bliebe die Bundesrepublik völkerrechtlich zur Umsetzung der entsprechenden Vertragsnormen verpflichtet. Dies würde bedeuten, dass Bund und Länder jeweils für ,ihre“ Arten des hoheitlichen Freiheitsentzuges gesetzliche Vorschriften schaffen müssten. Für den Bereich „Strafvollzug“ wären dann die Länder in der Pflicht.

Soll der präventive Ansatz der Europäischen und der Vereinte Nationen-Antifolterkonvention nicht relativiert werden, müsste de lege ferenda ein uneingeschränktes und unmittelbares Recht auf Akteneinsicht geschaffen werden. Der alternative Weg, die Gewährung der Akteneinsicht von einer - standardmäßig zum Vollzugsbeginn oder jeweils im Einzelfall einzuholenden - Einwilligung des Gefangenen abhängig zu machen, würde hingegen das Verfahren schwerfällig, unzuverlässig und missbrauchsanfällig machen. Es stünde der Bundesrepublik gut an, ihre Rechtspraxis und - falls dies als erforderlich angesehen wird - ihre Rechtslage im Sinne wirksamer Präventionsmechanismen vorbildlich auszugestalten.

118 So bspw.: § 26 Abs. 1 PolG NRW [Allgemeine Regeln der Datenübermittlung]: „Personenbezogene Daten dürfen nur zu dem Zweck übermittelt werden, zu dem sie erlangt oder gespeichert worden sind. Abweichend hiervon kann die Polizei personenbezogene Daten übermitteln, soweit dies 1. durch Gesetz zugelassen ist, 2. zur Abwehr einer Gefahr erforderlich ist und der Empfänger die Daten auf andere Weise nicht oder nicht rechtzeitig oder nur mit unverhältnismäßig hohem Aufwand erlangen kann. "§ 191 Abs. 1 S. 1 LVwG SH [Grundsätze der Datenübermittlung]: ,Personenbezogene Daten können nur zu dem Zweck übermittelt werden, zu dem sie erlangt oder gespeichert worden sind, soweit gesetzlich nichts anderes bestimmt ist.". 